\title{
Thrombopoietin signaling to chromatin elicits rapid and pervasive epigenome remodeling within poised chromatin architectures
}

\author{
Federico Comoglio, ${ }^{1,6,7}$ Hyun Jung Park, ${ }^{1,6}$ Stefan Schoenfelder, ${ }^{2}$ Iros Barozzi, ${ }^{3}$ \\ Daniel Bode, ${ }^{1}$ Peter Fraser, ${ }^{2,4}$ and Anthony R. Green ${ }^{1,5}$ \\ ${ }^{1}$ Cambridge Institute for Medical Research, Medical Research Council/Wellcome Trust Stem Cell Institute, and Department of \\ Haematology, University of Cambridge, Cambridge CB2 OXY, United Kingdom; ${ }^{2}$ Nuclear Dynamics Programme, The Babraham \\ Institute, Cambridge CB22 3AT, United Kingdom; ${ }^{3}$ Lawrence Berkeley National Laboratory, Berkeley, California 94720, USA; \\ ${ }^{4}$ Department of Biological Science, Florida State University, Tallahassee, Florida 32301, USA; ${ }^{5}$ Department of Haematology, \\ Addenbrooke's Hospital, Cambridge CB2 OXY, United Kingdom
}

\begin{abstract}
Thrombopoietin (TPO) is a critical cytokine regulating hematopoietic stem cell maintenance and differentiation into the megakaryocytic lineage. However, the transcriptional and chromatin dynamics elicited by TPO signaling are poorly understood. Here, we study the immediate early transcriptional and cis-regulatory responses to TPO in hematopoietic stem/ progenitor cells (HSPCs) and use this paradigm of cytokine signaling to chromatin to dissect the relationship between cisregulatory activity and chromatin architecture. We show that TPO profoundly alters the transcriptome of HSPCs, with key hematopoietic regulators being transcriptionally repressed within $30 \mathrm{~min}$ of TPO. By examining cis-regulatory dynamics and chromatin architectures, we demonstrate that these changes are accompanied by rapid and extensive epigenome remodeling of cis-regulatory landscapes that is spatially coordinated within topologically associating domains (TADs). Moreover, TPO-responsive enhancers are spatially clustered and engage in preferential homotypic intra- and inter-TAD interactions that are largely refractory to TPO signaling. By further examining the link between cis-regulatory dynamics and chromatin looping, we show that rapid modulation of cis-regulatory activity is largely independent of chromatin looping dynamics. Finally, we show that, although activated and repressed cis-regulatory elements share remarkably similar DNA sequence compositions, transcription factor binding patterns accurately predict rapid cis-regulatory responses to TPO.
\end{abstract}

[Supplemental material is available for this article.]

Hematopoiesis - the formation of blood cellular components-is an exquisitely characterized process in which the signaling consequences of extracellular stimuli, such as cytokines and growth factors, must be coherently integrated with chromatin structures to regulate cell-type-specific transcriptional programs (Rieger and Schroeder 2012). Specification of blood cell types is thought to proceed in a hierarchical fashion. At the apex of the hematopoietic hierarchy are multipotent hematopoietic stem cells (HSCs), which are able both to self-renew and to generate all blood cell lineages through differentiation into increasingly mature progenitor cells (Eaves 2015)

Lineage choice and commitment throughout hematopoiesis entails induction of lineage-specific gene regulatory networks and repression of lineage-inappropriate genes (Rieger and Schroeder 2012). These transcriptional programs are the result of coordinated waves of activation and decommissioning of unique constellations of cis-regulatory elements such as promoters and enhancers (Spitz and Furlong 2012). Cell-type-specific access to cisregulatory information reflects the combinatorial activity of transcription factors (TFs), which recruit chromatin modifiers to estab-

\footnotetext{
${ }^{6}$ These authors contributed equally to this work.

${ }^{7}$ Present address: Division of Gene Regulation, Netherlands Cancer Institute, Amsterdam, $1066 \mathrm{CX}$, The Netherlands

Corresponding authors: f.comoglio@nki.nl, arg1000@cam.ac.uk

Article published online before print. Article, supplemental material, and publication date are at http://www.genome.org/cgi/doi/10.1101/gr.227272.117. Freely available online through the Genome Research Open Access option.
}

lish active or repressive chromatin environments at regulatory elements within a cis-regulatory repertoire (Reiter et al. 2017).

Recent epigenomic advances have made it possible to map these repertoires genome-wide and across a wide range of cell types and organisms (The ENCODE Project Consortium 2012; Shlyueva et al. 2014). Putative enhancers can be defined as DNase I hypersensitive sites (DHSs) marked by monomethylated lysine 4 on histone $\mathrm{H} 3$ (H3K4me1), and their activity can be inferred based on the concomitant presence of histone $\mathrm{H} 3$ acetylation at lysine 27 (H3K27ac) often accompanied by detectable production of enhancer RNAs (eRNAs) (Heintzman et al. 2007, 2009; Creyghton et al. 2010; Calo and Wysocka 2013; Li et al. 2016). However, the targets of enhancers cannot be accurately predicted solely based on genomic proximity since transcriptional regulation occurs within the three-dimensional nuclear space (Bonev and Cavalli 2016; Dekker and Mirny 2016). Enhancers can exert their regulatory function on the expression of distally located target genes through three-dimensional chromatin looping that results in the juxtaposition of enhancer(s) and target promoter(s) (Bouwman and de Laat 2015; Bonev and Cavalli 2016). Experimental tethering of an enhancer to its target gene promoter has been shown to result in transcriptional activation of the $\mathrm{Hbb}$ locus, demonstrating that enhancer-promoter contacts can induce gene activation, even in the absence of a key transcriptional activator (Deng et al. 2012).

(C) 2018 Comoglio et al. This article, published in Genome Research, is available under a Creative Commons License (Attribution 4.0 International), as described at http://creativecommons.org/licenses/by/4.0/. 
Chromosome conformation capture (3C)-based methods, particularly high-throughput $3 \mathrm{C}(\mathrm{Hi}-\mathrm{C})$, have enabled targeted or genome-wide mapping of chromatin architectures (Denker and De Laat 2016). These technologies provided critical insights into key structural and functional components of three-dimensional chromatin organization such as (1) A/B compartments (Lieberman-Aiden et al. 2009), also referred to as compartment domains (Rao et al. 2017), which are closely associated with open and closed chromatin domains, respectively; (2) topologically associating domains (TADs) (Dixon et al. 2012; Nora et al. 2012; Sexton et al. 2012), also referred to as contact domains (Rao et al. 2017), chromosomal units that spatially constrain cis-regulatory interactions; and (3) CTCF loops, also referred to as insulated neighborhoods (Hnisz et al. 2016) or loop domains (Rao et al. 2017). Although these studies suggested a hierarchical domain organization, recent studies based on acute depletion of CTCF or cohesin, or inactivation of the cohesin-loading factor NIPBL, demonstrated that $\mathrm{A} / \mathrm{B}$ compartments and TADs are not hierarchically organized but represent independent structural (and possibly functional) units of 3D genome organization (Nora et al. 2017; Rao et al. 2017; Schwarzer et al. 2017; Wutz et al. 2017).

Enhancer-promoter communication preferentially occurs within TADs (Zhan et al. 2017). However, the high complexity of Hi-C libraries makes it impractical to systematically map cis-regulatory interactions with high resolution and coverage. To overcome this limitation, we recently developed Promoter Capture Hi-C (PCHi-C), a sequence capture approach that selectively enriches $\mathrm{Hi}-\mathrm{C}$ libraries for interactions involving more than 22,000 annotated mouse promoters, thus allowing global mapping of promoter interactions at restriction fragment level resolution (Schoenfelder et al. 2015).

Collectively, studies in different systems across multiple species using 3C-based methods and derivatives identified two types of enhancer-promoter interactions: loops formed de novo and preexisting loops (Montavon et al. 2011; Apostolou et al. 2013; Melo et al. 2013; Eijkelenboom et al. 2014; Ghavi-Helm et al. 2014; Cruz-Molina et al. 2017; Freire-Pritchett et al. 2017). Although de novo (also called instructive) interactions appear concomitant with changes in the target gene activity, preexisting (also called permissive) interactions are formed prior to gene activation and are thought to facilitate timely transcriptional induction (de Laat and Duboule 2013; Bouwman and de Laat 2015). However, the relationship between signal-dependent modulation of cis-regulatory activity and chromatin looping remains poorly understood for several reasons. First, poised chromatin architectures have been primarily studied in relation to gene activation, and little is known about signaling-dependent modulation of gene repression. Second, studies analyzing this relationship on developmental timescales lack the temporal resolution necessary to capture the early chromatin changes induced by signaling events. Third, physical interactions other than promoter-enhancer loops, such as enhancer-enhancer interactions, received comparatively little attention, albeit they likely represent an important layer of gene regulation in the three-dimensional nuclear space (Ing-Simmons et al. 2015).

The cytokine thrombopoietin (TPO) is a critical regulator of megakaryopoiesis. TPO regulates megakaryocyte and platelet production by activating its receptor, MPL (Bartley et al. 1994; de Sauvage et al. 1994; Lok et al. 1994), inducing multiple signaling pathways, including Janus kinase (JAK)/signal transducer and activator of transcription (STAT), mitogen-activated protein kinase, and phosphatidylinositol-3-kinase (De Graaf and Metcalf 2011).
The physiological role of TPO has been extensively studied in mouse knockout (KO) models. TPO KO mice exhibit severe thrombocytopenia with $90 \%$ reduction in platelet counts, indicating that TPO is the major physiological regulator of megakaryocyte and platelet production in vivo (de Sauvage et al. 1996). Moreover, TPO and $\mathrm{Mpl} \mathrm{KO}$ mouse models revealed that TPO signaling is also vital for hematopoietic stem cell maintenance and self-renewal (Kimura et al. 1998; Solar et al. 1998; Buza-Vidas et al. 2006; Qian et al. 2007; Yoshihara et al. 2007). However, these studies largely focused on TPO signaling at the level of cytoplasmic signaling pathways and/or cellular behavior. In contrast, the transcriptional consequences and cis-regulatory dynamics of TPO signaling to chromatin remain largely unknown.

\section{Results}

\section{The immediate early transcriptional response to TPO signaling}

To capture the immediate early transcriptional response to TPO, we performed subcellular RNA-seq (Bhatt et al. 2012) before and after 30-min TPO stimulation of HPC-7 cells, a cytokine-dependent and karyotypically normal multipotent hematopoietic stem/progenitor cell line (Pinto do Ó et al. 1998). HPC-7 cells self-renew in the presence of stem cell factor (SCF) and undergo megakaryocytic differentiation following TPO stimulation.

We isolated and profiled biochemically fractionated chromatin-associated, nucleoplasmic, and cytoplasmic transcripts in two biological replicates. Principal component analysis and hierarchical clustering indicate high reproducibility between replicates, with the subcellular compartment explaining most of the observed variation in gene expression (PC1, 72\%) (Supplemental Fig. S1A,B). As expected, this is largely driven by the high intronic content of chromatin-associated RNA-seq libraries, which are enriched for unprocessed and incompletely spliced transcripts (Supplemental Fig. S1C). In addition, analysis of small nuclear and small cytoplasmic RNA gene loci revealed compartment-specific transcript localization, confirming the high enrichment of our RNA fractions (Supplemental Fig. S1D).

We then analyzed the immediate early transcriptional consequences of TPO signaling at the chromatin level. We found that TPO transcriptionally up-regulated 1325 genes and repressed 639 genes (Fig. 1A). Importantly, significant changes in chromatin-associated RNA levels within 30 min of TPO stimulation only moderately overlapped changes detected in the cytoplasmic fraction ( $58 \%$ and $37 \%$ for up- and down-regulated genes, respectively) (Supplemental Fig. S1E) and exhibited significantly higher fold changes (Supplemental Fig. S1F). This suggests that RNA stability and post-transcriptional regulation contribute substantially to cytoplasmic gene expression changes even in response to transient signaling, underscoring the importance of profiling chromatin-associated or nascent transcripts when studying primary transcriptional responses. Unless otherwise stated, the remainder of our expression analyses focused on chromatin-associated transcripts.

The immediate early transcriptional consequences of TPO signaling to chromatin were highly divergent. On the one hand, genes most highly transcriptionally up-regulated by TPO included several targets of the canonical JAK/STAT signaling pathway (e.g., Cish, Pim1, Cited2, and Egr1) and the transcription factor MYC (Fig. $1 \mathrm{~A}, \mathrm{~B})$, suggesting broad housekeeping and survival functions. On the other hand, TPO led to the rapid transcriptional repression of key hematopoietic regulators such as HIf, Sox4, and Cxcr4 (Fig. 1B). Repression of these loci resulted in strongly decreased cytoplasmic

\section{Genome Research}

www.genome.org 
A

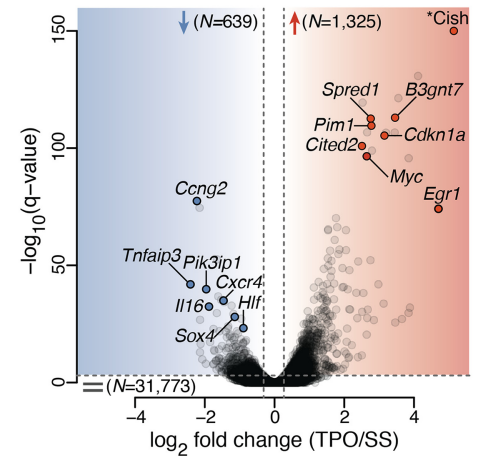

C

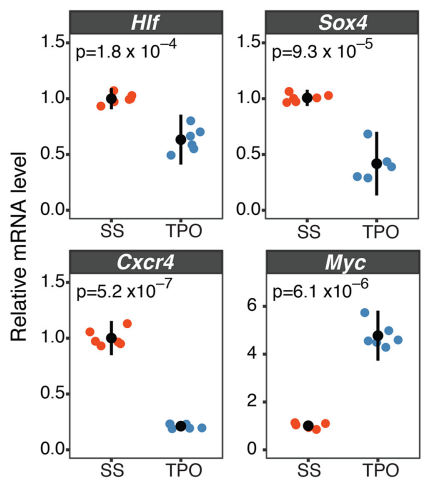

D

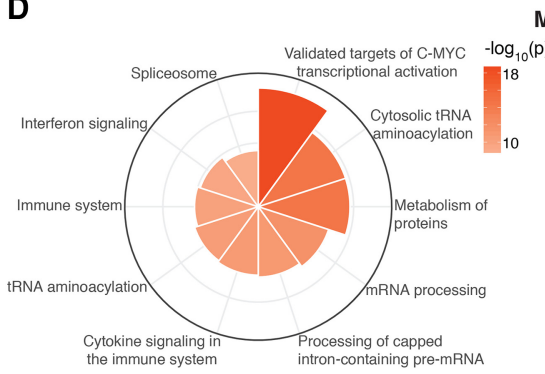

B

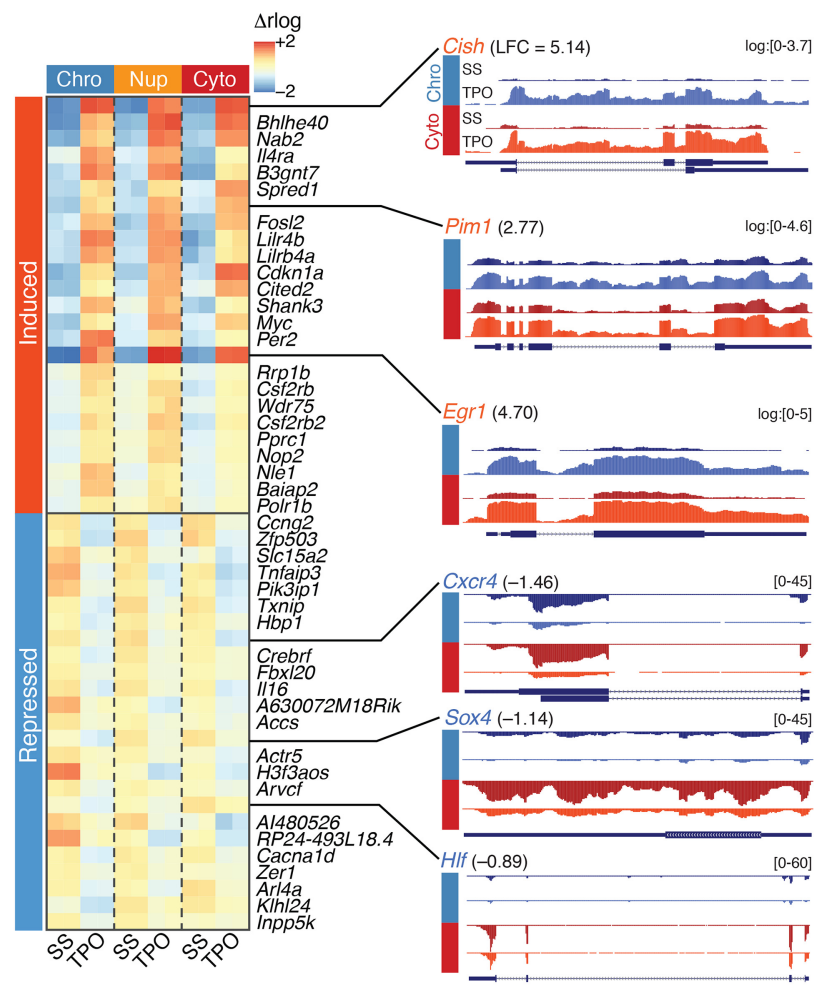

E

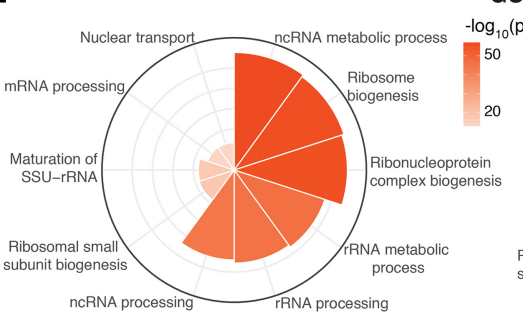

GO Biological process

SigDB pathways

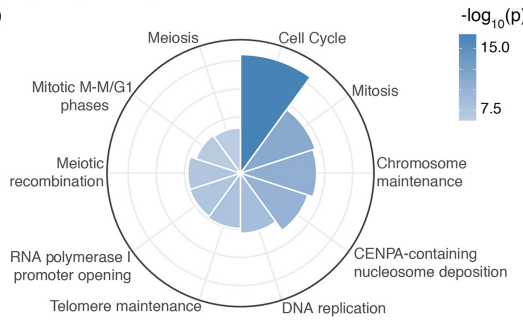

$\mathbf{F}$

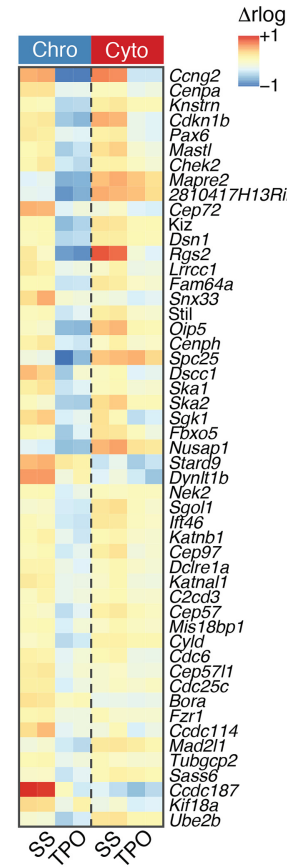

Figure 1. The immediate early transcriptional response to TPO signaling. ( $A$ ) Volcano plot of gene transcription changes induced by 30 min stimulation of HPC-7 cells with TPO relative to serum-starved (SS) control cells, based on chromatin-associated RNA expression. Red and blue shaded regions enclose transcriptionally up- and down-regulated genes $\left(Q-v a l u e ~<10^{-3}\right.$ ), respectively. The total number of genes within each category is indicated. Representative hits are labeled. The asterisk denotes the most strongly induced gene (Cish, $Q=1.8 \times 10^{-241}$ ), which was repositioned within the plot area. (B, left) Chromatin-associated (Chro), nucleoplasmic (Nup), and cytoplasmic (Cyto) RNA expression heatmap for the top 25 induced and top 25 repressed genes ranked by Q-value. Regularized $\log _{2}$ (rlog) expression values are row-mean subtracted. (Right) Representative tracks of differentially transcribed genes. Where indicated, RNA-seq coverage was log transformed with a pseudocount of 1 . (LFC) $\log _{2}$ fold change. (C) mRNA expression levels of the indicated genes in TPO-treated (30 min) primary CD41 ${ }^{+}$LSK cells, relative to serum-starved (SS) control cells, measured by quantitative RT-PCR. Error bars are mean $\pm \mathrm{SD}(n=6)$ from two mice. P-values are from a two-sided Welch's $t$-test. $(D)$ Top 10 significantly enriched Molecular Signature Database (MSigDB) pathways for transcriptionally up- (left) and down-regulated (right) genes, ranked by binomial $P$-value. (E) Same as $D$, for Gene Ontology (GO) biological process terms. $(F)$ Chromatin-associated (Chro) and cytoplasmic (Cyto) RNA expression heatmap of mitotic genes. Regularized log 2 (rlog) expression values are row-mean subtracted. 
RNA levels within 30 min of TPO, indicating that transcripts encoding these key regulators are subjected to rapid turnover. To validate our results in primary cells, we confirmed the rapid TPOinduced down-regulation of $H l f$, Sox 4 and Cxcr4, as well as up-regulation of $M y c$ by RT-qPCR in CD $41^{+} \mathrm{Lin}^{-}$ $\mathrm{Sca}^{+}{ }^{+} \mathrm{C}-\mathrm{Kit}^{+}\left(\mathrm{CD} 41^{+} \mathrm{LSK}\right)$ bone marrow cells (Fig. 1C; Nishikii et al. 2015), indicating that TPO elicits a common transcriptional program in megakaryocyticbiased hematopoietic progenitors.

To gain further insights into the nature of the transcriptional programs regulated by TPO, we subjected differentially transcribed genes to a Molecular Signatures Database (MSigDB) and Gene Ontology (GO) enrichment analyses. Transcriptionally up-regulated events were strongly enriched for housekeeping genes involved in RNA and protein metabolism, whose expression is largely driven by a MYC transcriptional program, and for genes that respond to cytokine signaling in the immune system (Fig. 1D,E). However, megakaryocytic-affiliated genes were not induced within 30 min of TPO (Supplemental Fig. S2), consistent with a slower induction kinetic (Park et al. 2015). In contrast, genes involved in mitosis, chromosome maintenance, and DNA repair were rapidly repressed by TPO (Fig. 1D-F). A distinctive feature of megakaryopoiesis is endomitosis, DNA replication in the absence of cell division that results from an incomplete $\mathrm{M}$ phase due to a failure in late cytokinesis (Bluteau et al. 2009). The rapid transcriptional repression of genes involved in mitotic nuclear division and microtubule organization (Fig. 1F) suggests that a cell cycle switch to endomitosis might occur very rapidly at the transcriptional level. Alternatively, it might represent a more general phenomenon of cell differentiation (Ruijtenberg and van den Heuvel 2016).

TPO signaling elicits rapid and extensive epigenome remodeling of cis-regulatory landscapes

Next, we set out to investigate the chromatin dynamics underlying the immediate early response to TPO by surveying the activity of cis-regulatory elements before and after $30 \mathrm{~min}$ TPO stimulation of HPC-7 cells. To this end, we profiled H3K27ac genome-wide by chromatin immunoprecipitation sequencing (ChIPseq) in two biological replicates and used a sliding window-based approach to detect genomic regions exhibiting significantly altered H3K27ac levels at $1 \%$

\section{C}

E false discovery rate (FDR) (Fig. 2A; Lun and Smyth 2015; Methods).

Our analysis identified 10,222 differentially acetylated regions (DARs) exhibiting significantly increased (Gain, $n=2669$ )
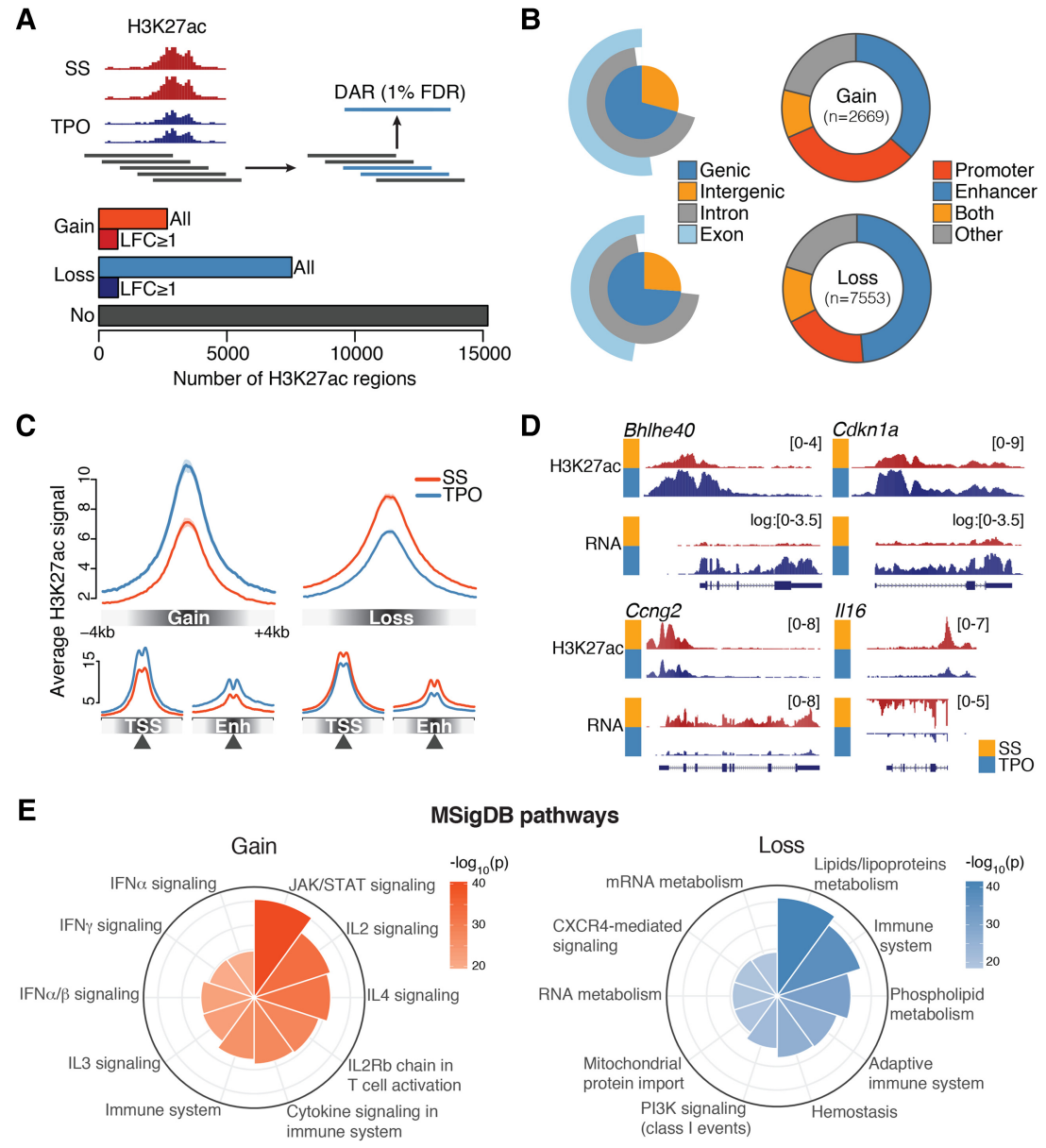

$\mathbf{F}$

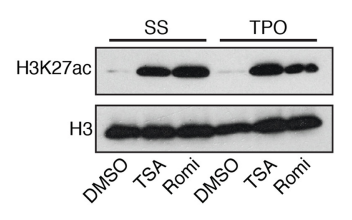

G

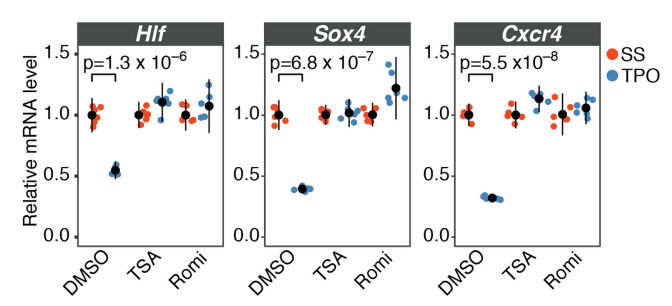

Figure 2. TPO signaling elicits rapid and extensive epigenome remodeling at cis-regulatory elements. $(A, t o p)$ Schematic representation of the tiling-window approach used to identify differentially acetylated regions (DARs) from H3K27ac ChIP-seq profiles. Significant overlapping windows (blue rectangles) were merged to call DARs at $1 \%$ false discovery rate (FDR). (Bottom) Total number of activated (Gain) and repressed (Loss) DARs. H3K27ac regions not significantly altered by $30 \mathrm{~min}$ TPO (No) are also shown. (LFC) $\log _{2}$ fold change. ( $B$, left) Annotation of DARs with respect to genomic compartments. (Right) Annotation of DARs with respect to cis-regulatory elements inferred from DNase-seq profiles in serum-starved HPC-7 cells (Methods). (C) Average normalized H3K27ac signal within $\pm 4 \mathrm{~kb}$ of DAR summits (top), transcription start sites (TSSs), or enhancer summits (Enh). (D) Representative tracks of DARs. Where indicated, chromatin-associated RNA-seq coverage was log transformed with a pseudocount of 1 . (E) Genomic regions enrichment of annotations tool (GREAT) analysis of DARs. Top 10 significantly enriched Molecular Signature Database (MSigDB) pathways for gene associated with DARs, ranked by binomial $P$-value. $(F)$ Western blot analysis of H3K27ac and total H3 levels in HPC-7 cells pretreated for 30 min with DMSO, Trichostatin A (TSA), or Romidepsin (Romi), before and after 30 min TPO stimulation. (G) Corresponding mRNA expression levels of the indicated genes relative to serum-starved (SS) control cells, measured by quantitative RT-PCR. Error bars are mean \pm SD $(n=6)$ from two biological replicates. P-values are from a two-sided Welch's $t$-test.

\section{Genome Research}

www.genome.org 
or decreased (Loss, $n=7553$ ) H3K27ac levels within 30 min of TPO treatment (Fig. 2A). This number represents a sizeable fraction (40\%) of genomic regions marked by H3K27ac in basal condition, demonstrating widespread (albeit not global) changes of cis-regulatory activity in response to transient cytokine signaling (Fig. 2A). Activated and repressed DARs showed overlapping size distributions with a median length of $3.4 \mathrm{~kb}$ (Supplemental Fig. S3A). However, the two sets differed in the magnitude of their response to TPO, with activated DARs exhibiting significantly stronger changes in H3K27ac levels (Fig. 2A; Supplemental Fig. S3B). We ruled out the possibility that these broad acetylation changes could stem from technical biases, because H3K27ac promoter signals at the 1000 most highly expressed genes unaffected by TPO were highly correlated $(r=0.94-0.99)$ and exhibited no systematic difference between replicates and conditions (Supplemental Fig. S3C). In addition, changes in the abundance of enhancer RNA (eRNA) transcripts at differentially acetylated intergenic enhancers correlated with changes in H3K27ac levels, further validating our DAR calls (Supplemental Fig. S3D). Moreover, to rule out the possibility that differential acetylation events reflect global changes in H3 occupancy, we measured H3K27ac and total H3 levels at a set of differentially acetylated loci by ChIP-qPCR (Supplemental Fig. $\mathrm{S} 3 \mathrm{E})$. This analysis indicates that H3K27ac changes at DARs represent bona fide acetylation dynamics.

We next mapped DARs onto genomic compartments. Less than $30 \%$ of DARs were intergenic, with the majority of acetylation changes overlapping annotated genes (Fig. 2B). To more precisely localize cis-regulatory elements within DARs, we mapped active promoters and enhancers at high resolution using DNaseseq profiles in self-renewing HPC-7 cells (Wilson et al. 2016; Methods). The vast majority (80\%) of DARs encompassed elements (DHSs) from these two regulatory classes (Fig. 2B-D).

To investigate the functional significance of DARs, we performed a Genomic Regions Enrichment of Annotations Tool (GREAT) analysis by linking DARs to their putative target genes (McLean et al. 2010). As expected, activated DARs were strongly linked with genes involved in cytokine and JAK/STAT signaling (Fig. 2E). In contrast, repressed DARs were significantly associated to genes involved in lipid metabolism and in the regulation of the immune system, particularly of adaptive immunity (Fig. 2E), indicating that TPO signaling represses the cis-regulatory elements of lymphoid-affiliated genes.

Finally, to test whether deacetylation of cis-regulatory elements is required for transcriptional repression of key hematopoietic regulators (Fig. 1B,C), we pretreated HPC-7 cells with DMSO or the HDAC inhibitors Trichostatin A (TSA) or Romidepsin (Romi) for $30 \mathrm{~min}$ (Fig. 2F) and measured gene expression changes induced by $30-$ min TPO stimulation by RT-qPCR. We found that whereas $\mathrm{Hlf}$, Sox4, and Cxcr4 were significantly down-regulated within $30 \mathrm{~min}$ of TPO treatment in DMSO-treated cells, TSA or Romi fully abrogated this response (Fig. 2G).

Together, these results demonstrate that TPO signaling elicits rapid and extensive epigenome remodeling at cis-regulatory elements, and histone deacetylation is necessary for transcriptional repression of key hematopoietic regulators.

\section{Cis-regulatory dynamics induced by TPO signaling are spatially coordinated within topological domains}

We noted that homotypic DARs tended to strongly cluster along the chromatin fiber, suggesting spatial coordination within chromatin domains (Fig. 3A). Thus, we set out to systematically inves- tigate how the rapid modulation of cis-regulatory activity induced by TPO is spatially coordinated within the nucleus. To this end, we generated Hi-C libraries from HPC-7 cells before and after 30-min TPO stimulation in two biological replicates and identified valid di-tags using the Hi-C Pro pipeline (Servant et al. 2015). We obtained a of total of 644 and 675 million valid di-tags in basal and TPO-treated conditions, respectively. Of these, $565(87.7 \%)$ and $593(87.9 \%)$ million valid di-tags, respectively, were intrachromosomal paired-end reads.

We first focused on megabase-sized chromatin domains. We found that higher-order chromatin structures, including A/B compartments, TAD boundary location and strength, and CTCF loops, were largely unaffected by transient TPO signaling (Supplemental Fig. S4A-F). This result is in line with previous studies of extracellular signaling that examined higher-order chromatin architectures $1 \mathrm{~h}$ after stimulation in other cellular systems (Jin et al. 2013; Le Dily et al. 2014).

We then analyzed the distribution of DARs within TADs identified in basal condition. This analysis revealed a clear spatial segregation of homotypic TPO-responsive DARs within topological domains (Fig. 3B), indicating that changes in cis-regulatory activity induced by TPO are spatially correlated at the level of TADs. To more formally investigate this aspect, we identified TADs exhibiting significantly altered $\mathrm{H} 3 \mathrm{~K} 27$ ac levels in response to TPO at $1 \%$ FDR (Fig. 3C; Methods). This analysis singled out 200 (6\% of all H3K27ac-marked TADs) globally induced and 535 (16\%) globally repressed TADs (henceforth collectively called differentially acetylated TADs), allowing us to study topological domains that were mostly perturbed by TPO.

Compared to TADs with no significant changes in H3K27ac, differentially acetylated TADs were moderately enriched (approximately twofold) for differentially transcribed genes. As expected, genes in repressed TADs showed an overall lower expression than genes in activated TADs (Fig. 3D). Notably, although only $3 \%$ of down-regulated genes were located in activated TADs, a much higher fraction (12.9\%) of up-regulated genes lay within repressed TADs (Fig. 3E). Thus, whereas down-regulated genes were virtually excluded from activated TADs, repressed TADs appeared at least partially permissive to transcriptional up-regulation (Fig. 3E).

Finally, we examined the relationship between transcriptional and cis-regulatory responses to TPO by correlating the number of differentially transcribed genes with the number of DARs located within the same differentially acetylated TAD. As expected, we found a marked correlation between increased cis-regulatory activity and transcriptional up-regulation (Fig. 3F). In contrast, we noticed a weaker association between repressed cis-regulatory elements and transcriptionally down-regulated genes (Fig. 3F). To further explore this aspect, we analyzed the distribution of differentially transcribed genes within all TADs exhibiting at least two homotypic DARs. To our surprise, we found that most TADs with repressed DARs did not show a corresponding detectable repression of genes located therein (Supplemental Fig. S4G), thus explaining the relatively weak association between cis-regulatory and transcriptional repression. Importantly, basal expression levels of these genes were significantly lower than for genes located within TADs hosting at least one differentially transcribed gene (Supplemental Fig. S4H). The lower expression levels reduce our ability to detect statistically significant further reductions in transcript levels. However, our results raise the possibility that repressed DARs within these loci might represent the first detectable step toward stable gene silencing of lineage-inappropriate genes. 
A

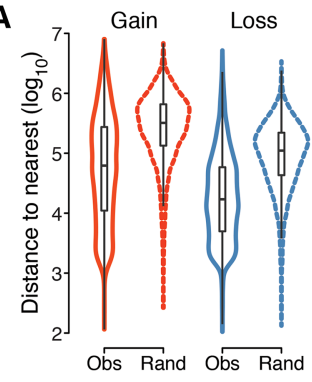

C

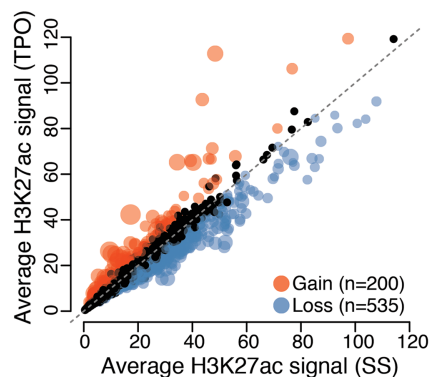

B
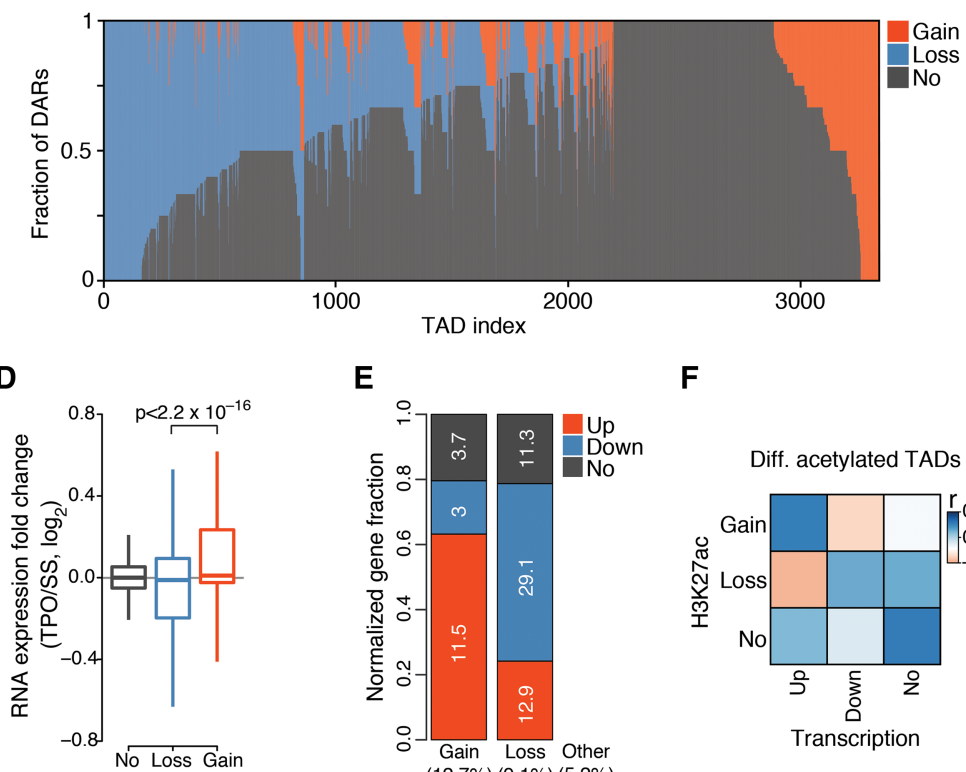

E

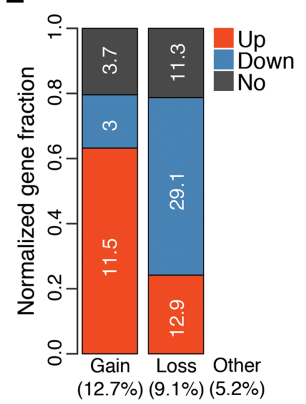

F

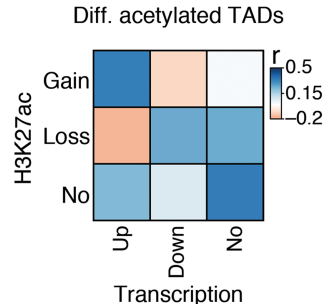

Diff. acetylated TADs

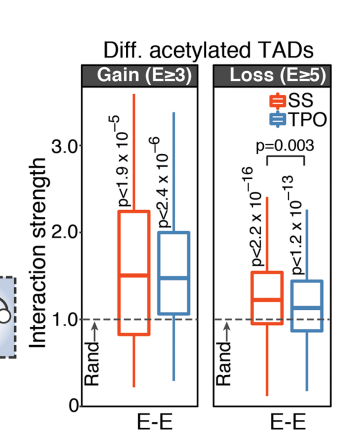

G

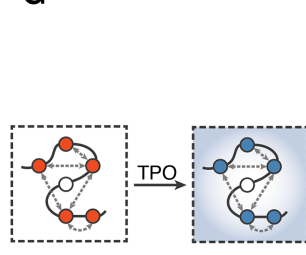

Diff. acetylated TADs

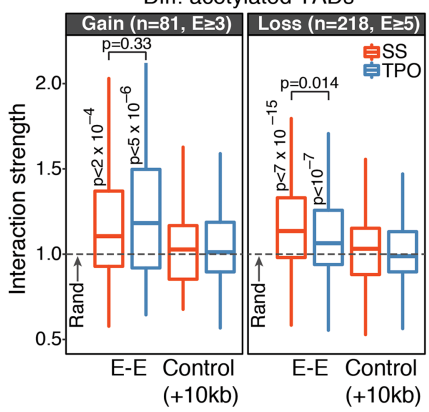

H

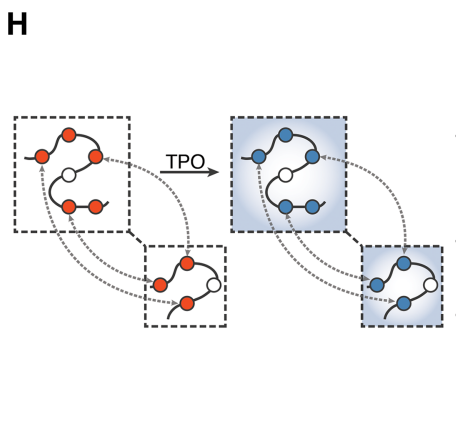

Figure 3. Cis-regulatory responses to TPO are spatially coordinated within the nucleus. ( $A$ ) Distribution of observed (Obs) and expected (Rand) (Methods) genomic distances between nearest homotypic DARs. (B) Distribution of DARs within TADs detected in serum-starved HPC-7 cells and containing at least one H3K27ac region. Each TAD corresponds to a vertical line. (C) Average normalized H3K27ac signals per kilobase of TAD. Significantly differentially acetylated TADs are highlighted. Point sizes are proportional to - $\log _{10}(Q$-value). (D) Distribution of chromatin-associated RNA expression fold changes of genes localized within differentially acetylated TADs. $P$-value is from a Wilcoxon rank-sum test. $(E)$ Relative fraction of differentially transcribed genes localized within differentially acetylated TADs, normalized by total number of genes within each category. The percentage of genes in each category is indicated inside the bar plot, whereas the percentage of differentially transcribed genes falling within each TAD class is indicated at the bottom. $(F)$ Spearman's rank correlation matrix between frequency of DAR and frequency of differentially transcribed genes within differentially acetylated TADs. (G) Structured interaction matrix analysis (SIMA) of enhancer-enhancer interactions for differentially acetylated enhancers within differentially acetylated TADs. The interaction strength reflects the enrichment of $\mathrm{Hi}-\mathrm{C}$ interactions relative to randomly sampled genomic regions. Interaction strength distributions for matched controls (Methods) are also shown. P-values are from a Wilcoxon signed rank test (for testing differences from random interactions) and from a Wilcoxon rank-sum test (for comparison between conditions). (H) Same as $G$, for enhancer-enhancer interactions between differentially acetylated TADs located within $20 \mathrm{Mb}$ blocks.

\section{TPO-responsive enhancers are spatially clustered}

At finer scales, physical interactions between promoter-distal sites appear to be widespread (Fullwood et al. 2009; Phillips-Cremins et al. 2013; Ghavi-Helm et al. 2014; Sahlén et al. 2015) and might function to provide specificity and robustness to enhancer-promoter interactions within cis-regulatory units (MarkenscoffPapadimitriou et al. 2014; Ing-Simmons et al. 2015). Previous work suggests that enhancer elements tend to cluster in the nuclear space in a cohesin-dependent manner (Ing-Simmons et al. 2015), but how enhancer-enhancer interactions are modulated by extracellular signaling remains largely unknown.

To investigate this aspect, we took advantage of the extensive and spatially compartmentalized epigenome remodeling induced by transient TPO signaling and analyzed enhancer-enhancer interactions within and between differentially acetylated TADs using a structured interaction matrix analysis (SIMA) (Lin et al. 2012). This method pools Hi-C interactions across a predefined set of genomic regions (in our case, enhancer elements) and computes their interaction strength relative to control regions randomly sampled from the same set of chromatin domains (differentially acetylated TADs). First, we focused on enhancer-enhancer interactions within activated and repressed TADs. In the absence of TPO, we found that homotypic enhancers interacted significantly more frequently with each other than expected based on a null model (Fig. 3G), indicating that they tend to congregate within topological domains. Importantly, this was not the case when enhancer coordinates were systematically shifted by $10 \mathrm{~kb}$ along the

\section{Genome Research}

www.genome.org 
chromatin fiber, demonstrating that our analysis is well calibrated (Fig. 3G). We then examined the consequences of TPO signaling. Enhancer-enhancer interactions were only moderately perturbed within $30 \mathrm{~min}$ of TPO (Fig. 3G). Indeed, homotypic enhancers remained significantly clustered within differentially acetylated TADs, suggesting that TPO selectively modulates enhancer-enhancer interactions rather than altering them at a global scale.

Although by definition intra-TAD interactions occur more frequently than interactions spanning TAD boundaries, topological domains represent a modest twofold enrichment in interaction frequency (Dixon et al. 2012; Nora et al. 2012; Sexton et al. 2012). Therefore, we tested whether enhancers located within neighboring TPO-regulated TADs show evidence of spatial clustering (Methods). We found that, similarly to intra-TAD interactions, inter-TAD enhancer-enhancer interactions were significantly enriched over random expectation and only moderately perturbed by transient TPO signaling (Fig. 3H). This enrichment was further confirmed by an analysis of enhancer-enhancer interactions between CTCF loops (Supplemental Fig. S3F).

Together, these results indicate that TPO-responsive enhancers engage in preferential long-range intra- and inter-TAD interactions resulting in their clustering in the three-dimensional nuclear space, and TPO-induced changes in enhancer activity are at least partly uncoupled from this spatial clustering of enhancer elements.

\section{TPO-responsive super-enhancers control the expression of key hematopoietic regulators}

Transcriptional enhancers not only interact with each other through long-range interactions but also within dense enhancer hotspots known as super-enhancers (SEs) or stretch-enhancers (Parker et al. 2013; Whyte et al. 2013; Ing-Simmons et al. 2015), which have been implicated in the transcriptional control of cell identity genes (Hnisz et al. 2013). Intriguingly, a recent study revealed that SE-associated genes are enriched for functional categories relevant to cytokine biology in mouse $\mathrm{T}$ lymphocytes (Vahedi et al. 2015). Therefore, we reasoned that SEs might be prime candidates for the integration of TPO signaling to chromatin.

To test this hypothesis, we identified SEs in serum-starved HPC-7 cells (Fig. 4A; Methods) and analyzed their response to 30-min TPO stimulation. TPO signaling significantly perturbed the activity of more than half of all SEs (162 of 277, 58\%) identified in basal condition. The vast majority of these TPO-responsive SEs (140 of $162,86 \%$ ) were significantly deacetylated within 30 min of TPO, indicating that TPO signaling primarily represses SE activity (Fig. 4B; Supplemental Fig. S5A,B). We next examined the consequences of this perturbation on the transcription of putative target genes as a function of their genomic distance to SEs. Differential acetylation of SEs was more frequently linked to transcriptional perturbation of SE-proximal genes located within genomic distances $<1 \mathrm{Mb}$ (Fig. 4C; Supplemental Fig. S5C), with 27\% of activated SEs and $22 \%$ of repressed SEs localizing within the same CTCF loop as their closest differentially transcribed gene. Nevertheless, the majority of genes located in close proximity to TPO-responsive SEs exhibited little or no detectable transcriptional changes within 30 min of TPO (Fig. 4C). This result suggests that SE chromatin dynamics might often precede transcriptional changes at target genes or that genomic proximity is not an accurate predictor of SE targets.
To more accurately link SEs to their target genes, we enriched our Hi-C libraries for interactions anchored at 22,225 annotated gene promoters within the mouse genome using Promoter Capture Hi-C (PCHi-C) (Schoenfelder et al. 2015), for a total of 445 and 407 million intrachromosomal valid di-tags in basal and TPO-treated conditions, respectively. Data normalization and interaction detection from two biological replicates using the CHiCAGO pipeline (Cairns et al. 2016) resulted in 192,634 and 181,235 statistically significant (CHiCAGO score $\geq 5$ ) promoteranchored long-range cis-interactions for basal and TPO-treated conditions, respectively. Focusing on SEs, we found that approximately $2.4 \%$ of all significant PCHi-C interactions linked promoters to SE constituents (Fig. 4D), allowing us to infer the targets of differentially acetylated SEs based on spatial proximity. Although most SE-interacting genes showed no detectable transcriptional changes within $30 \mathrm{~min}$ of TPO, we found that targets of induced and repressed SEs were enriched for transcriptionally up- and down-regulated genes, respectively, and these included known SE targets such as Myc and Etv6 (Fig. 4E,F; Khan and Zhang 2016). Promoters of key hematopoietic regulators rapidly repressed by TPO signaling (Hlf, Sox4, and Cxcr4) (Fig. 1B,C) were highly connected to constituent enhancers within repressed SEs (Fig. 4F), suggesting that their expression is controlled from a distance through TPO-responsive SEs.

\section{Rapid modulation of cis-regulatory activity is largely independent of chromatin looping dynamics}

Next, we tested whether the pervasive epigenome remodeling observed within differentially acetylated TADs and within SEs was accompanied by systematic changes in chromatin looping. To this end, we computed normalized Hi-C contact frequencies within these chromatin domains before and after 30-min TPO stimulation. Our analysis revealed no systematic differences in contact frequencies between conditions, which exhibited nearly perfectly correlated Hi-C signals ( $r>0.99)$ (Fig. 5A). This was further confirmed by a systematic analysis of chromatin interactions anchored at DARs (Fig. 5A). These results indicate that, despite substantial changes in both transcriptome and epigenome, chromatin architectures at differentially acetylated TADs and SEs were essentially unaltered following TPO stimulation.

We then focused on the spatial chromatin architecture at differentially transcribed gene loci, taking advantage of the increased resolution offered by PCHi-C over Hi-C data. We found that most promoters of differentially transcribed genes engaged more than 10 promoter-interacting regions (PIRs) (with a median of 13 and 14 PIRs for transcriptionally up- and down-regulated genes, respectively) (Fig. 5B); although for transcriptionally up-regulated genes these interactions tended to span significantly shorter genomic distances (Fig. 5C), no major differences were found in the connectivity of these loci. In addition, $63 \%$ of all promoter interactions detected by PCHi-C were anchored at H3K27ac-marked regions (Fig. 5D), with $30.7 \%$ encompassing at least one DAR, indicating extensive coverage of these genomic regions.

These results prompted us to systematically correlate changes in $\mathrm{H} 3 \mathrm{~K} 27 \mathrm{ac}$ levels with changes in PCHi-C interaction frequency within individual differentially transcribed cis-regulatory units (Methods). For each promoter, we defined a cis-regulatory unit as the union of all its H3K27ac-marked PIRs, and we set a threshold of at least five such regions being required to ensure robust estimates of H3K27ac fold changes and correlation coefficients. This resulted in a set of 907 differentially transcribed loci (Fig. 5E). 
A

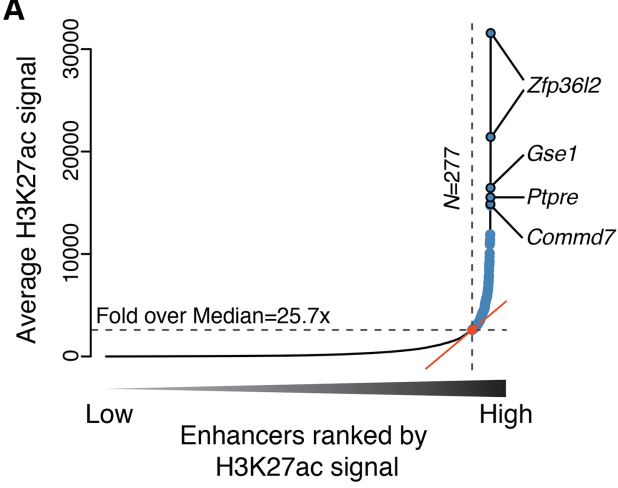

B

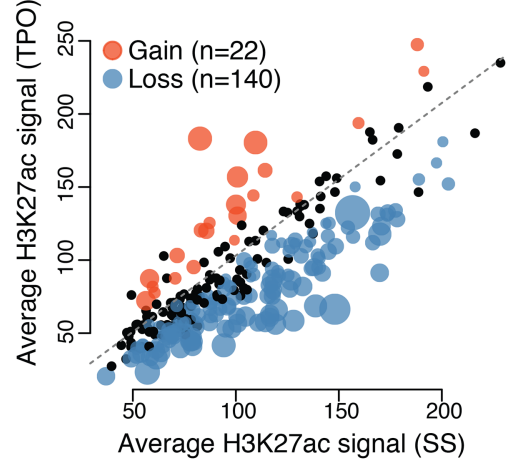

E

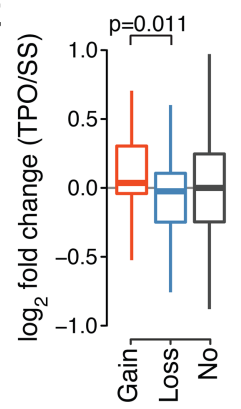

웡
C

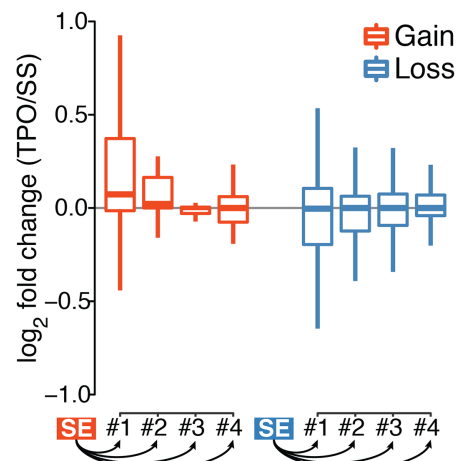

D

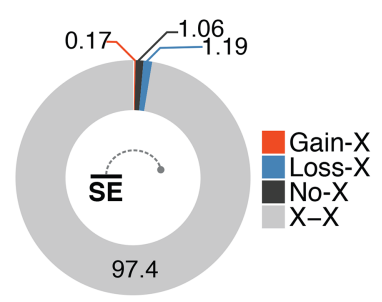

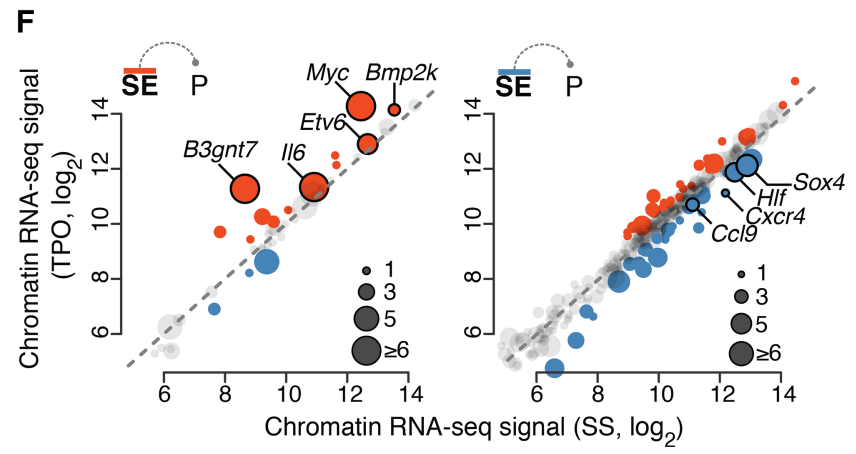

Figure 4. TPO signaling is integrated at super-enhancers. ( $A$ ) Distribution of average (control-subtracted) normalized H3K27ac ChIP-seq signals at enhancer regions in serum starved HPC-7 cells. Super-enhancers (SEs, blue) exhibit exceptionally high H3K27ac levels. (B) Average normalized H3K27ac signal per kilobase of SE. Significantly differentially acetylated SEs are highlighted. Point sizes are proportional to -log $10\left(Q-v_{1}\right.$ alue). (C, top) Distribution of chromatin-associated RNA expression fold change for first, second, third, and fourth nearest gene to a differentially acetylated SE. (Bottom) Corresponding distribution of genomic distances. (D) Percentage of significant promoter Capture Hi-C interactions anchored at SEs. (X) any HindIII restriction fragment located outside SEs. (E) Distribution of chromatin-associated RNA expression fold changes of SE-target genes defined by promoter Capture Hi-C. $P$-value is from a Wilcoxon rank-sum test. $(F)$ Chromatin-associated RNA expression (regularized $\log _{2}$ values) of gene targets of differentially acetylated SE as defined in $E$. Point sizes are proportional to the number of constituent enhancers exhibiting significant interactions with the gene promoter. Selected SE targets are labeled.

We found that the vast majority of cis-regulatory units exhibited little or no correlation between changes in cis-regulatory activity and chromatin looping (Fig. 5E).

This result was further supported by a permutation test in which the connectivity of cis-regulatory units was randomly scrambled to derive a null distribution for the correlation coefficients (Methods). Our data revealed that only $5.4 \%$ of differentially transcribed gene loci being tested (49 of 907, corresponding to $4.8 \%$ and $6.7 \%$ of analyzed transcriptionally up- and down-regulated genes) exhibited a statistically significant correlation between acetylation and looping dynamics in response to transient TPO signaling (Fig. 5E). Neither rapidly induced genes (e.g., Myc and Spred1) (Fig. 1A) nor repressed loci encoding key hematopoietic regulators belonged to this set (Fig. 5F). Indeed, although these loci exhibited extensive differential acetylation within $30 \mathrm{~min}$ of
TPO, this was not accompanied by a rewiring of chromatin loops (Fig. 5F,G; Supplemental Fig. S6).

Together, these results demonstrate that the extensive and spatially compartmentalized epigenome remodeling induced by transient TPO signaling takes place within poised chromatin architectures and suggest that the rapid cytokine-dependent modulation of cis-regulatory activity is largely independent of chromatin looping dynamics.

TF binding patterns but not DNA sequence features accurately predict rapid cis-regulatory responses to TPO

Finally, we asked which TFs orchestrate the rapid cis-regulatory dynamics induced by TPO signaling. To identify TFs that control activated and repressed cis-regulatory elements within DARs, we 
A

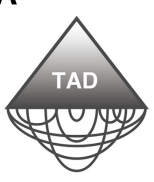

SE

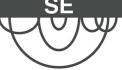

DAR $\stackrel{>20 \mathrm{~kb}}{\longrightarrow}$
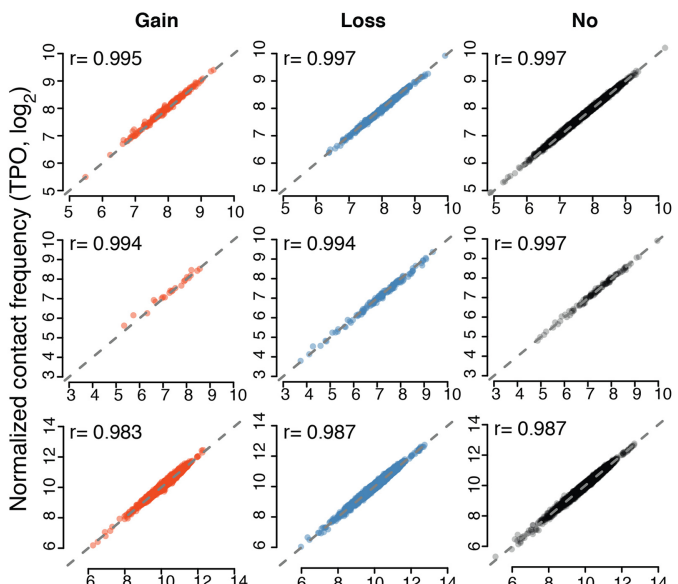

Normalized contact frequency (SS, $\log _{2}$ )

E
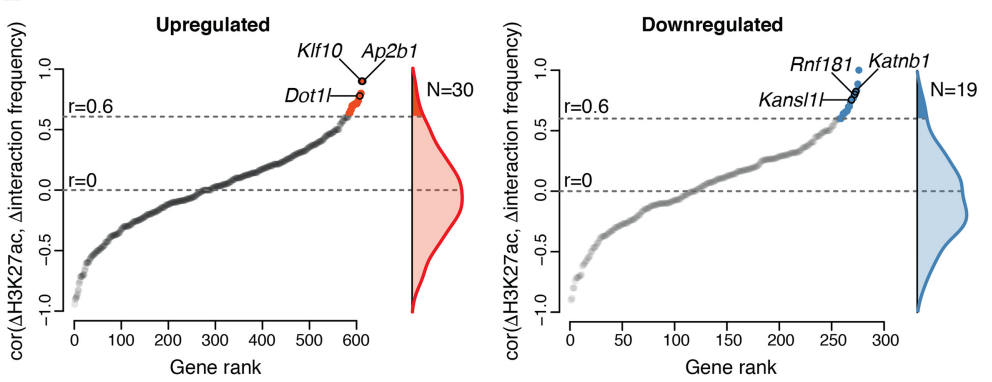
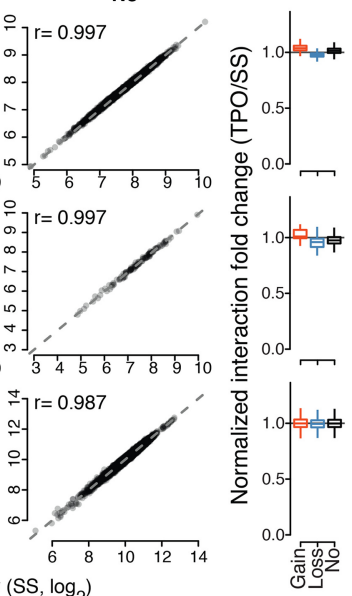

D
B

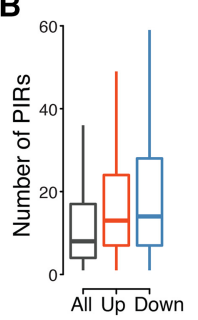

C

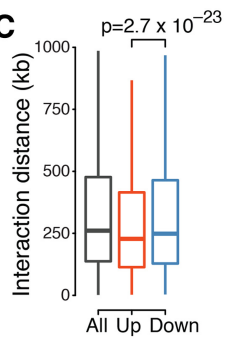

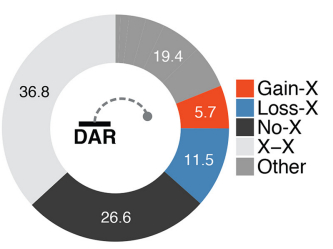

$\mathbf{F}$

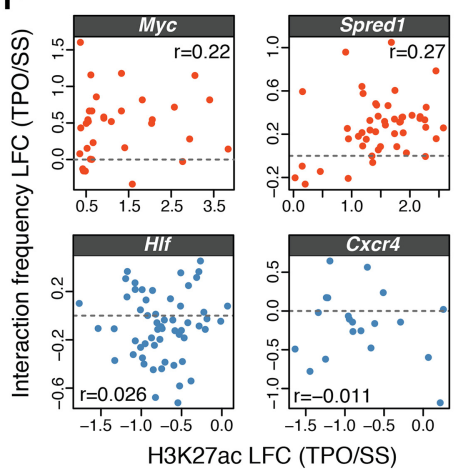

G

Chr11:88,037,971-92,146,829(mm10)
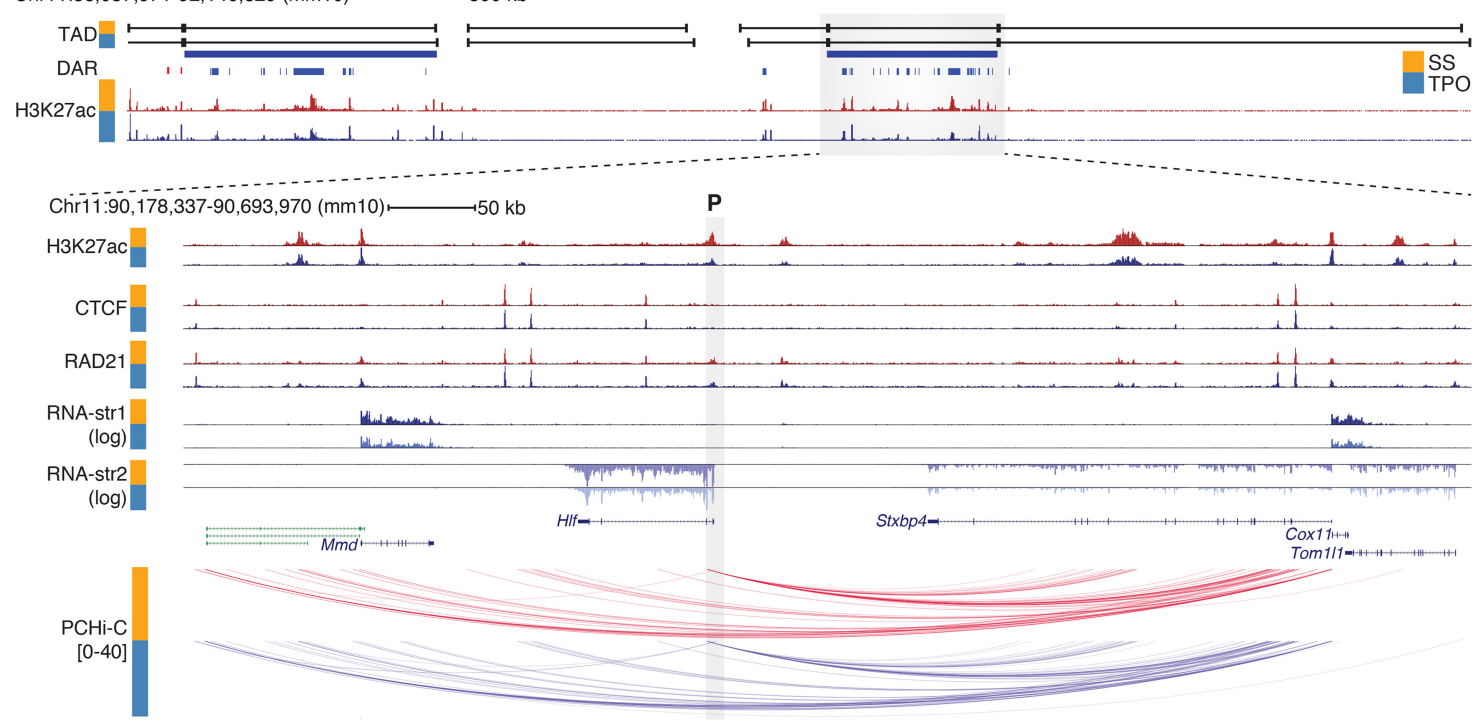

Figure 5. Rapid modulation of cis-regulatory activities within poised chromatin architectures. $(A)$ Normalized $\mathrm{Hi}-\mathrm{C}$ contact frequencies for intra-TAD and intra-SE interactions (per kilobase of element), and for interactions anchored at DARs spanning more than $20 \mathrm{~kb}$. Spearman's rank correlation coefficients $(r)$ are shown. Box plots (right) summarize normalized interaction fold change distributions. (B) Distribution of the number of promoter-interacting regions (PIRs) for all baited promoters (All) and promoters of transcriptionally up- and down-regulated genes. (C) Distribution of interaction distances for all baited promoters (All) and promoters of transcriptionally up- and down-regulated genes. P-value is from a Wilcoxon rank-sum test. (D) Percentage of significant promoter Capture Hi-C interactions anchored at DARs. $(X)$ any Hindlll restriction fragment located outside DARs. (E) Relationship between cis-regulatory activity and chromatin architectures at cis-regulatory units for transcriptionally up- and down-regulated genes (Methods). Genes are ranked based on the Spearman's rank correlation coefficient $(r)$ between normalized H3K27ac fold change (TPO/SS) and normalized Capture Hi-C interaction fold change at target DARs. Only promoters exhibiting significant interactions with at least five distinct DAR-containing Hindlll restriction fragments were considered. Genes exhibiting significant correlations are colored, and representative hits are labeled. $(F)$ Representative examples from the analysis in $E$. Each dot corresponds to an HindllI restriction fragment. (LFC) $\log _{2}$ fold change. (G) Epigenomic configuration of the HIf locus. Statistically significant promoter Capture Hi-C interactions within the HIf TAD are shown. The gray shaded rectangle denotes the position of the baited Hlf promoter (P). (str) strand. 
subjected differentially acetylated DHSs to a de novo motif discovery analysis. We found that both activated and repressed DARs were strongly enriched for ETS motifs, which feature a central $5^{\prime}$ GGAA-3' core, and for motifs recognized by the RUNX and GATA families of TFs (Fig. 6A,B). These motifs exhibited similar enrichments and overlapping distributions at activated and repressed DARs (Fig. 6B). In stark contrast, an interferon $\gamma$-activation sequence (GAS) motif, which is recognized by tyrosine-phosphorylated STAT (pSTAT) TFs, was specifically overrepresented at activated but not at repressed DARs (Fig. 6B), indicating that canonical JAK/STAT signaling is exclusively associated with DAR activation. Surprisingly, however, a systematic motif enrichment analysis based on 363 position weight matrices (PWMs) for 322 vertebrate TFs revealed that no TF motif other than those recognized by pSTATs exhibited a comparably specific association with either DAR class (Fig. 6C). Thus, despite exhibiting opposite response to TPO, activated and repressed cis-regulatory elements were characterized by a remarkably similar TF motif composition.

This result led us to perform an unbiased survey of DNA and chromatin features that might be able to predict rapid cis-regulatory responses to TPO signaling. To this end, we considered activated and repressed cis-regulatory elements and trained least absolute shrinkage and selection operator (lasso) logistic regression models (Tibshirani 1996) using different sets of features (Fig. 6D; Methods). These included DNA sequence $k$-mers $(2 \leq$ $k \leq 4)$, DNA shape features (Chiu et al. 2016), a collection of more than 1700 single and composite TF motifs (Diaferia et al. 2016) that was filtered for TFs expressed in HPC-7 cells, a collection of 29 ChIP-seq binding profiles for hematopoietic and other sequence-specific TFs in HPC-7 cells before $(n=26)$ and after $(n=3)$ 30-min TPO stimulation (generated as part of this study or previously published) (Wilson et al. 2010, 2016; Park et al. 2015; Supplemental Table S1), and normalized Hi-C signals as a proxy for interaction frequencies. Model performances were evaluated on a test set consisting of differentially acetylated cis-regulatory elements that were not used for learning model parameters (Fig. 6D).

Although DNA sequence features were only moderately predictive for cis-regulatory responses to TPO (area under the receiver operating characteristic curve, AUC $<0.70$ ), in vivo TF binding profiles were able to accurately predict changes in cis-regulatory activity $(\mathrm{AUC}=0.80)$ (Fig. 6E). In contrast, Hi-C signals showed virtually no predictive power $(\mathrm{AUC}=0.53$ ) (Fig. 6E), further supporting the notion that these changes occur within poised chromatin architectures. In addition, more complex models combining sequence and chromatin features did not outperform models learned on TF binding profiles alone (Supplemental Fig. S7A), suggesting that key TFs were already included within our chromatin feature set. Furthermore, these results were validated by an independent learning scheme using random forest classifiers (Supplemental Fig. S7B; Breiman 2001).

Next, we focused on the models learned on TF binding profiles and sought to identify the chromatin features that contribute the most to model predictions by estimating feature stability coefficients using bootstrap-Lasso (Comoglio and Paro 2014; Comoglio et al. 2015). Intuitively, the more a feature is necessary for accurate predictions, the higher its stability value. Our analysis revealed that preexisting MYC binding and binding of pSTAT1 upon TPO stimulation were stably associated with the activation of cis-regulatory elements (Fig. 6F; Supplemental Fig. S7C). In contrast, binding of MYB, LYL1, and tyrosine unphosphorylated STAT5 (uSTAT5) in the absence of TPO was predictive for repression of cis-regulatory elements (Fig. 6F; Supplemental Fig. S7C).
Notably, DARs bound by stably selected features were significantly associated with distinct signaling pathways and metabolic functions, spanning the whole spectrum of biological processes regulated by TPO (Fig. 6G).

We previously demonstrated that in the absence of TPO, chromatin-bound uSTAT5 restrains a megakaryocytic transcriptional program in hematopoietic stem/progenitor cells, and TPOmediated phosphorylation of STAT5 triggers a genome-wide relocation of STAT5 to canonical GAS motifs (Park et al. 2015). Intriguingly, uSTAT5 knockdown not only up-regulated megakaryocytic-affiliated genes, but it also repressed lineage-inappropriate genes including lymphoid- and pregranulocyte/monocyte-affiliated genes (Park et al. 2015). This result, along with our feature importance analysis (Fig. 6F), led us to hypothesize that uSTAT5 binding might be required to maintain the activity of cis-regulatory elements within gene loci rapidly repressed by TPO. To test this hypothesis, we took advantage of a mutant (Y699F) STAT5B that ablates TPO-induced tyrosine phosphorylation of STAT5 and does not act as a dominant negative (Park et al. 2015). We expressed wild-type or mutant STAT5B in HPC-7 cells and measured H3K27ac levels at uSTAT5-bound enhancers within the HIf, Sox4, and Cxcr4 loci (Fig. 6H) by ChIP-qPCR before and after 30-min TPO stimulation. The Y699F mutant significantly attenuated deacetylation at uSTAT5-bound enhancers in response to TPO compared to wild type (Fig. 6I), indicating that uSTAT5 binding is required to maintain the activity of these elements.

\section{Discussion}

In this study, we focused on the immediate early consequences of TPO signaling to chromatin and examined the relationship between signal-dependent modulation of cis-regulatory activity and chromatin looping. Our work provides three key findings.

First, we present evidence for rapid and pervasive changes in cis-regulatory activity that are readily detectable within $30 \mathrm{~min}$ of TPO. Interestingly, this widespread epigenome remodeling encompasses up to $40 \%$ of active genomic regions marked by H3K27ac prior to TPO stimulation, indicating that short-term cytokine signaling is sufficient to profoundly alter chromatin states at cis-regulatory elements. Notably, a sizeable fraction of these remodeling events corresponds to histone deacetylation of enhancer elements associated with genes playing key roles in innate and adaptive immune cells. This rapid deacetylation of enhancer elements likely represents the first step toward decommissioning of active enhancers associated with lineage-inappropriate transcriptional programs (Smith and Shilatifard 2014) and suggests that TPO signaling rapidly dismantles cis-regulatory networks affiliated to alternative lineages.

Second, we demonstrate that TPO-induced modulation of cisregulatory units occur largely independently of alterations in chromatin looping, indicating that a responsiveness to TPO is hardwired into a poised HSPC spatial genome architecture prior to cytokine exposure. It is important to note, however, that TPO-dependent modulation of short-range interactions between enhancers and promoters would not be detectable in our PCHi-C data.

A previous Hi-C analysis of chromatin structures after 1-h TNF stimulation of primary human fibroblast cells showed that TNF-responsive enhancers are already in contact with their target promoters prior to stimulation, and these preexisting chromatin structures appear to be strong predictor of gene induction (Jin et al. 2013). Similarly, preformed chromatin loops have also been detected at inducible gene loci regulated by TP53, FOXO3,

\section{Genome Research}

www.genome.org 
A

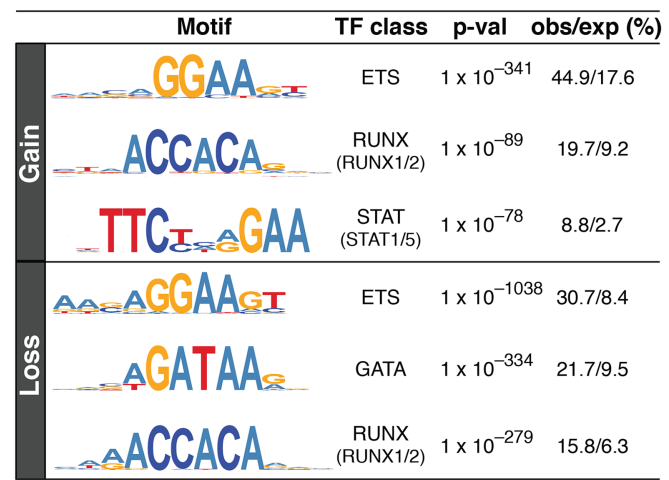

D

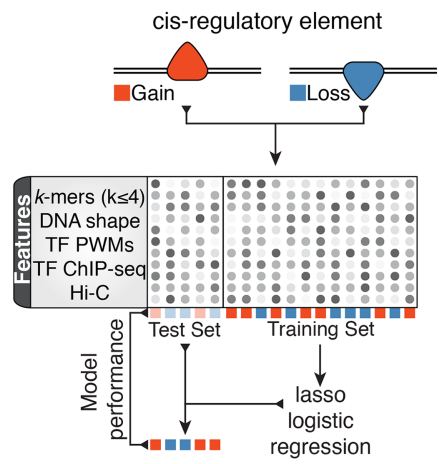

G

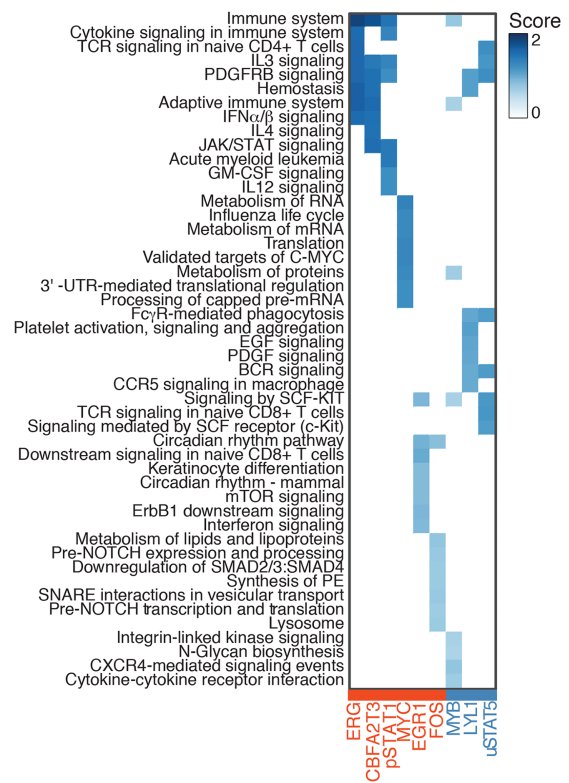

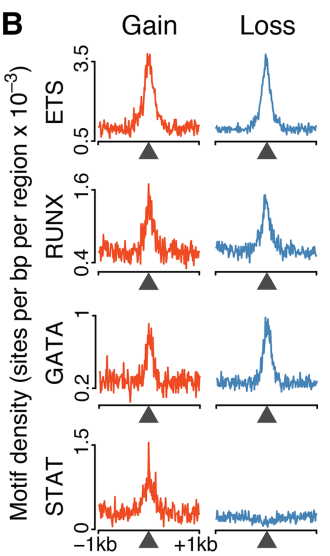

E

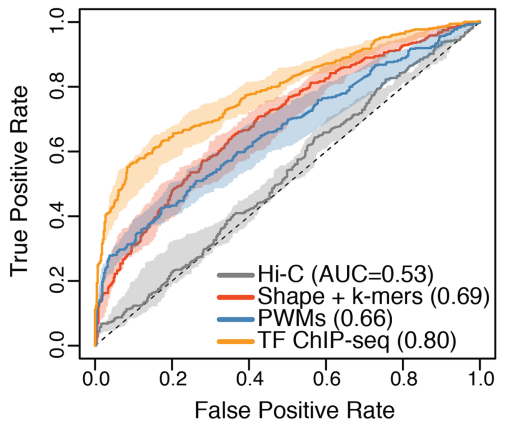

H

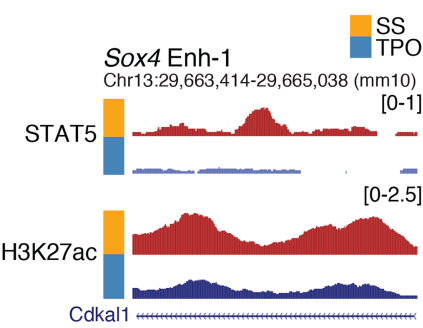

Cxcr4 Enh

Chr1:128,704,623-128,705,842 (mm10)

STAT5

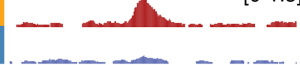

H3K27ac

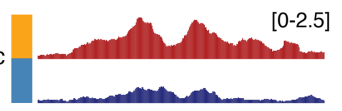

C

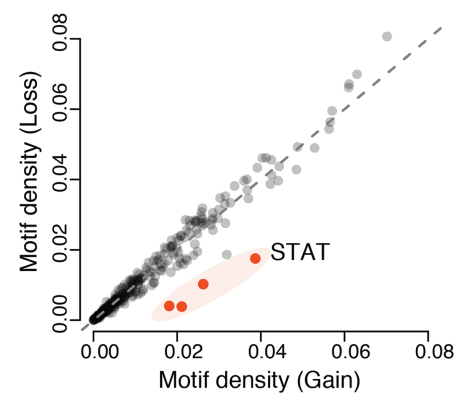

F

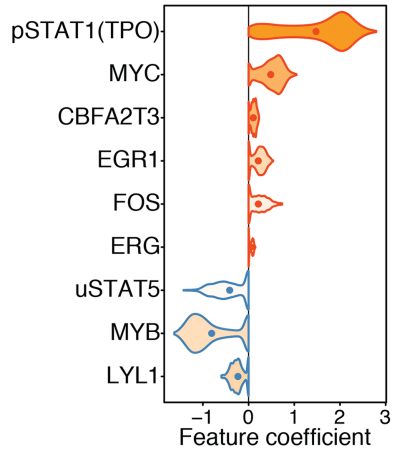

I

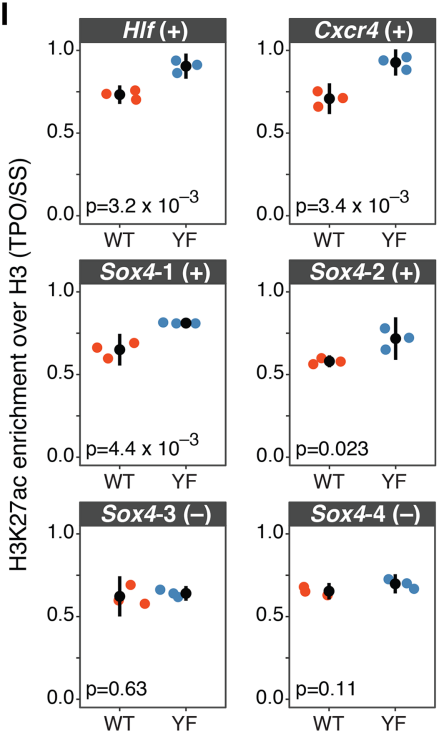

Figure 6. TF binding patterns accurately predict rapid cis-regulatory responses to TPO. ( $A$ ) Top transcription factor motifs (position weight matrices [PWMs]) identified by de novo motif discovery analysis within $200 \mathrm{nt}$ of the summit of differentially acetylated DHSs (Methods). The percentage of observed and expected motif occurrence is indicated. (B) Motif density (PWMs) for the indicated transcription factor motifs within $\pm 1 \mathrm{~kb}$ of the summit of differentially acetylated DHSs. (C) Total motif density within $200 \mathrm{nt}$ of the summit of differentially acetylated DHSs, for a collection of 363 vertebrate transcription factor PWMs. (D) Schematic outline of the statistical learning strategy used to predict how cis-regulatory elements within DARs respond to TPO. (E) Test set receiver operating characteristic (ROC) curve and area under the ROC curve (AUC) values for lasso models trained on the indicated sets of features. The shaded area is delimited by the ROC curves for models with highest and lowest AUC values, whereas the ROC curve for the model closest to mean AUC is shown. $(F)$ Top-ranked features selected by bootstrap-Lasso for the model trained on TF ChIP-seq profiles, ranked by selection probability (stability). Violin plot lines are color-coded according to coefficient signs: (red) positive; (blue) negative. (G) Genomic regions enrichment of annotations tool (GREAT) analysis of differentially acetylated cis-regulatory elements bound by TFs in F. The top eight significantly enriched Molecular Signature Database (MSigDB) pathways were considered for each transcription factor, ranked by binomial $P$-value. $(H)$ Representative tracks illustrating STAT5 binding dynamics at differentially acetylated enhancers within key hematopoietic gene loci. (I) Relative H3K27ac enrichment (TPO/SS, normalized to total H3 levels) at the indicated enhancer elements (USTAT5-bound [+] or uSTAT5 negative [-, control]) for HPC-7 cells expressing wild-type (WT) or mutant (Y699F) STAT5B. Tested loci include the enhancers shown in $H$. Error bars are mean \pm SD $(n=3)$. P-values are from a two-sided Welch's $t$-test. 
and hormone receptor binding by $3 \mathrm{C}$ or circular chromosome conformation capture (4C) sequencing (Jin et al. 2013; Melo et al. 2013; Eijkelenboom et al. 2014). Our results indicate that poised chromatin architectures not only provide permissive regulatory topologies for transcriptional induction, but also a platform for rapid signaling-dependent repression of cis-regulatory units. Therefore, it is plausible that disruption of preformed chromatin architectures prior to TPO stimulation might similarly impair both activation and repression of cis-regulatory units in response to TPO signaling. However, further work will be required to formally test this hypothesis.

Third, we show that TPO signaling can induce opposite responses at cis-regulatory elements exhibiting markedly similar DNA sequence compositions and regulatory codes. This unexpected finding raises the question about which molecular determinants direct these opposing events. Regulatory instructions are encoded in DNA sequences by the identity, frequency, affinity, and grammar of TF binding sites. However, how these parameters dictate cis-regulatory dynamics is poorly understood (Spitz and Furlong 2012; Levo and Segal 2014). Our results indicate that, with the notable exception of pSTATs, the mere presence or absence of binding motifs for specific TFs cannot predict individual cis-regulatory responses to TPO and raise the possibility that orientation and spacing of TF motifs might play a role in determining these responses. Consistent with this concept, a recent work demonstrated that a different motif grammar based on the same building blocks defines activating and repressing cis-regulatory elements in rod photoreceptors (White et al. 2016). Moreover, several studies showed that a subset of TFs can act both as repressor or activator depending on cellular states, sequence contexts, and binding of cofactors (Méthot and Basler 1999; Sharrocks 2001; Nayak et al. 2009; Hollenhorst et al. 2011; Liu et al. 2014; Sánchez-Tilló et al. 2015). Deciphering the motif grammar that distinguishes activated and repressed cis-regulatory elements should be an important goal of future studies. To this end, statistical learning models considering motif orientation and spacing could be used to guide the design of massively parallel reporter assays based on synthetic regulatory elements or more targeted in vitro assays (Inoue and Ahituv 2015; Fiore and Cohen 2016).

Although our analysis suggests that regulatory priming in multipotent progenitors does not appear to be constrained by sequence composition, in vivo TF binding patterns are able to accurately predict rapid cis-regulatory responses to TPO. Interestingly, feature importance analysis indicates that preexisting TF binding prior to TPO stimulation significantly contributes to these predictions. This notion is further supported by the targeted experimental analysis of USTAT5-bound enhancers, where preexisting uSTAT5 binding is predictive for the repression of these regulatory elements. Moreover, our statistical models identified other potential key players mediating rapid cis-regulatory responses to TPO such as MYB and LYL1. These hematopoietic TFs are critically involved in the maintenance of HSPCs as well as in lymphoid differentiation (Capron et al. 2006; Fahl et al. 2009; Lieu and Reddy 2009; Souroullas et al. 2009; Greig et al. 2010). Our results suggest that MYB and LYL1 binding prior to TPO stimulation predicts deacetylation of cis-regulatory elements associated with the lymphoid lineage. Therefore, it is tempting to speculate that TPO supports megakaryocytic lineage specification in part by restricting the developmental potential of multipotent progenitor cells. To this end, it would be interesting to further investigate whether TPO directly regulates chromatin binding and/or activity of MYB and LYL1 in HSPCs.
Taken together, our study unravels the multifaceted immediate early consequences of TPO signaling to chromatin and provides a paradigm for the integrative epigenomic analysis of cytokine signaling.

\section{Methods}

\section{Cells and cell culture}

HPC-7 cells were grown at $37^{\circ} \mathrm{C}$ and $5 \% \mathrm{CO}_{2}$ in IMDM (Invitrogen) supplemented with $10 \%$ fetal calf serum, $10 \%$ SCF conditioned media (produced by the BHK/MKL cell line), 1\% L-Glutamine, $1 \%$ penicillin/streptomycin, and $74.8 \mu \mathrm{M}$ monothioglycerol (Sigma). Cell density was maintained between $5 \times 10^{5}$ and $2 \times$ $10^{6}$ cells $/ \mathrm{mL}$.

\section{Mice}

Eight- to twelve-week-old C57BL/6 mice (Charles River) were used in this study. All mouse procedures were approved by the UK Home Office and the University of Cambridge Animal Welfare and Ethical Review Board.

\section{Serum starvation and cytokine stimulation}

Cells were spun down at $1000 \mathrm{rpm}$ for $5 \mathrm{~min}$ and washed once in PBS. The cell pellet was resuspended at a density of $1 \times 10^{6}$ cells $/ \mathrm{mL}$ in StemSpan SFEM medium (StemCell Technologies) and incubated for $4 \mathrm{~h}$. Serum-starved cells were then stimulated with recombinant murine thrombopoietin (TPO, Peprotech) at $100 \mathrm{ng} / \mathrm{mL}$ for 30 min. TPO was diluted in a small volume of fresh StemSpan SFEM medium prior to stimulation.

\section{Isolation of $\mathrm{CD} 41^{+} \mathrm{LSK}$ cells}

Freshly isolated mouse bone marrow cells were stained with AF700-conjugated lineage cocktail (133313), APC-Cy7-conjugated anti-cKit (105826), Bv605-conjugated anti-Sca1 (108133), and FITC-conjugated anti-CD41 (133903). 7-AAD (Invitrogen) was used to exclude dead cells. All antibodies were purchased from BioLegend unless otherwise indicated. Cells were sorted on fluorescence-activated cell sorting (FACS) Aria (BD Bioscience) directly into StemSpan SFEM medium (StemCell Technologies). The sorted cells were incubated for $1 \mathrm{~h}$ at $37^{\circ} \mathrm{C}$ and $5 \% \mathrm{CO}_{2}$ prior to stimulation with TPO as described above.

\section{HDAC inhibitor treatment}

HPC-7 cells were starved as described above. Cells were pretreated with HDAC inhibitors, $1 \mu \mathrm{M}$ of Trichostatin A, or $5 \mathrm{nM}$ of Romidepsin (Selleckchem) during the final 30 min of serum starvation and stimulated with TPO at $100 \mathrm{ng} / \mathrm{mL}$ for $30 \mathrm{~min}$.

\section{STAT5 expression}

Retrovirus was produced using Phoenix cells (ATCC). HPC-7 cells were infected with retrovirus expressing STAT5B or mutant (Y699F) STAT5B in the presence $8 \mu \mathrm{g} / \mathrm{mL}$ polybrene (Millipore). After $24 \mathrm{~h}$, GFP-positive cells were FACS sorted and cultured in IMDM media (Invitrogen) supplemented with 10\% fetal bovine serum and $10 \%$ SCF conditioned media.

\section{RNA preparation and RT-qPCR}

Total RNA was isolated using Direct-zol (Zymo Research) and reverse transcribed into cDNA using SuperScript IV First Strand Synthesis System (Invitrogen) following the manufacturer's

\section{Genome Research}

www.genome.org 
instructions. Quantitative RT-PCR was carried out using SYBR Green Brilliant II low Rox and a Stratagene Mx3000P machine (Agilent Technologies). For primer sequences, see Supplemental Table S2.

\section{Subcellular RNA isolation}

Subcellular fractionation and RNA preparation were performed essentially as described (Bhatt et al. 2012) with modifications detailed in Supplemental Methods.

\section{Chromatin immunoprecipitation}

Chromatin immunoprecipitation (ChIP) assays on serum-starved and TPO-stimulated HPC-7 cells were performed as previously described (Supplemental Methods; Wilson et al. 2010).

\section{$\mathrm{Hi}-\mathrm{C}$}

Hi-C libraries were generated essentially as described (Schoenfelder et al. 2015) with modifications detailed in Supplemental Methods.

\section{Promoter Capture Hi-C}

Promoter Capture Hi-C libraries were generated essentially as described (Schoenfelder et al. 2015) with modifications detailed in Supplemental Methods.

\section{Computational analyses}

Subcellular RNA-seq, ChIP-seq, Hi-C, and promoter Capture Hi-C data processing and all computational analyses are detailed in Supplemental Methods.

\section{Data access}

RNA-seq, ChIP-seq, Hi-C, and promoter Capture Hi-C raw and processed data from this study have been submitted to the NCBI Gene Expression Omnibus (GEO; http://www.ncbi.nlm.nih.gov/geo/) under accession number GSE100835.

\section{Acknowledgments}

We thank Bertie Göttgens for providing access to raw data prior to their publication, and David Flores-Santa-Cruz and Rebecca Hannah for technical support. We also thank Gioacchino Natoli, Chiara Balestrieri, and Liv Austenaa (European Institute of Oncology) for help with subcellular RNA isolation and TF motif analysis, and Luca Giorgetti and Zhan Yinxiu (Friedrich Miescher Institute) for discussion. F.C. was supported by an EMBO longterm fellowship (1305-2015 and Marie Curie Actions LTFCOFUND2013/GA-2013-609409). Work in the Fraser laboratory was supported by the UK Biotechnology and Biological Sciences Research Council (Grant Ref. BB/J004480/1). Work in the Green laboratory was supported by Bloodwise (Grant Ref. 13003), the Wellcome Trust (Grant Ref. 104710/Z/14/Z), the Medical Research Council, the Kay Kendall Leukaemia Fund, the Cambridge NIHR Biomedical Research Center, the Cambridge Experimental Cancer Medicine Centre, the Leukemia and Lymphoma Society of America (Grant Ref. 07037), and a core support grant from the Wellcome Trust and MRC.

Author contributions: F.C. and H.J.P. conceptualized the study; F.C., H.J.P., and S.S. designed the methodology; F.C., I.B., and D.B. carried out the formal analysis; F.C., H.J.P., and S.S. conducted the investigation; P.F. and A.R.G. acquired the resources; F.C. wrote the original draft; F.C., H.J.P., S.S., I.B., P.F., and A.R.G. contributed to writing, reviewing, and editing; F.C. was responsible for visual- ization; S.S., P.F., and A.R.G. supervised; P.F. and A.R.G. acquired funding.

\section{References}

Apostolou E, Ferrari F, Walsh RM, Bar-Nur O, Stadtfeld M, Cheloufi S, Stuart HT, Polo JM, Ohsumi TK, Borowsky ML, et al. 2013. Genome-wide chromatin interactions of the Nanog locus in pluripotency, differentiation, and reprogramming. Cell Stem Cell 12: 699-712.

Bartley TD, Bogenberger J, Hunt P, Li YS, Lu HS, Martin F, Chang MS, Sama B, Nichol JL, Swift S, et al. 1994. Identification and cloning of a megakaryocyte growth and development factor that is a ligand for the cytokine receptor MpI. Cell 77: 1117-1124.

Bhatt DM, Pandya-Jones A, Tong AJ, Barozzi I, Lissner MM, Natoli G, Black DL, Smale ST. 2012. Transcript dynamics of proinflammatory genes revealed by sequence analysis of subcellular RNA fractions. Cell 150: 279-290.

Bluteau D, Lordier L, Di Stefano A, Chang Y, Raslova H, Debili N, Vainchenker W. 2009. Regulation of megakaryocyte maturation and platelet formation. J Thromb Haemost 7: 227-234.

Bonev B, Cavalli G. 2016. Organization and function of the 3D genome. Nat Rev Genet 17: 772 .

Bouwman BA, de Laat W. 2015. Getting the genome in shape: the formation of loops, domains and compartments. Genome Biol 16: 154.

Breiman L. 2001. Random forests. Mach Learn 45: 5-32.

Buza-Vidas N, Antonchuk J, Qian H, Månsson R, Luc S, Zandi S, Anderson K, Takaki S, Nygren JM, Jensen CT, et al. 2006. Cytokines regulate postnatal hematopoietic stem cell expansion: opposing roles of thrombopoietin and LNK. Genes Dev 20: 2018-2023.

Cairns J, Freire-Pritchett P, Wingett SW, Várnai C, Dimond A, Plagnol V, Zerbino D, Schoenfelder S, Javierre BM, Osborne C, et al. 2016. CHiCAGO: robust detection of DNA looping interactions in Capture Hi-C data. Genome Biol 17: 127.

Calo E, Wysocka J. 2013. Modification of enhancer chromatin: what, how, and why? Mol Cell 49: 825-837.

Capron C, Lécluse Y, Kaushik AL, Foudi A, Lacout C, Sekkai D, Godin I, Albagli O, Poullion I, Svinartchouk F, et al. 2006. The SCL relative $L Y L-1$ is required for fetal and adult hematopoietic stem cell function and B-cell differentiation. Blood 107: 4678-4687.

Chiu TP, Comoglio F, Zhou T, Yang L, Paro R, Rohs R. 2016. DNAshapeR: an $\mathrm{R} /$ Bioconductor package for DNA shape prediction and feature encoding. Bioinformatics 32: 1211-1213.

Comoglio F, Paro R. 2014. Combinatorial modeling of chromatin features quantitatively predicts DNA replication timing in Drosophila. PLoS Comput Biol 10: e1003419.

Comoglio F, Schlumpf T, Schmid V, Rohs R, Beisel C, Paro R. 2015. Highresolution profiling of Drosophila replication start sites reveals a DNA shape and chromatin signature of metazoan origins. Cell Rep 11: 821-834.

Creyghton MP, Cheng AW, Welstead GG, Kooistra T, Carey BW, Steine EJ, Hanna J, Lodato MA, Frampton GM, Sharp PA, et al. 2010. Histone H3K27ac separates active from poised enhancers and predicts developmental state. Proc Natl Acad Sci 107: 21931-21936.

Cruz-Molina S, Respuela P, Tebartz C, Kolovos P, Nikolic M, Fueyo R, van Ijcken WFJ, Grosveld F, Frommolt P, Bazzi H, et al. 2017. PRC2 facilitates the regulatory topology required for poised enhancer function during pluripotent stem cell differentiation. Cell Stem Cell 20: 689-705.

De Graaf CA, Metcalf D. 2011. Thrombopoietin and hematopoietic stem cells. Cell Cycle 10: 1582-1589.

de Laat W, Duboule D. 2013. Topology of mammalian developmental enhancers and their regulatory landscapes. Nature 502: 499-506.

de Sauvage FJ, Hass PE, Spencer SD, Malloy BE, Gurney AL, Spencer SA, Darbonne WC, Henzel WJ, Wong SC, Kuang WJ, et al. 1994. Stimulation of megakaryocytopoiesis and thrombopoiesis by the cMpl ligand. Nature 369: 533-538.

de Sauvage FJ, Carver-Moore K, Luoh SM, Ryan A, Dowd M, Eaton DL, Moore MW. 1996. Physiological regulation of early and late stages of megakaryocytopoiesis by thrombopoietin. J Exp Med 183: 651656.

Dekker J, Mirny L. 2016. The 3D genome as moderator of chromosomal communication. Cell 164: 1110-1121.

Deng W, Lee J, Wang H, Miller J, Reik A, Gregory PD, Dean A. 2012. Controlling long-range genomic interactions at a native locus by targeted tethering of a looping factor. Cell 149: 1233-1244.

Denker A, De Laat W. 2016. The second decade of 3C technologies: detailed insights into nuclear organization. Genes Dev 30: 1357-1382.

Diaferia GR, Balestrieri C, Prosperini E, Nicoli P, Spaggiari P, Zerbi A, Natoli G. 2016. Dissection of transcriptional and cis-regulatory control of differentiation in human pancreatic cancer. EMBO J 35: 595-617. 
Dixon JR, Selvaraj S, Yue F, Kim A, Li Y, Shen Y, Hu M, Liu JS, Ren B. 2012. Topological domains in mammalian genomes identified by analysis of chromatin interactions. Nature 485: 376-380.

Eaves C. 2015. Hematopoietic stem cells: concepts, definitions, and the new reality. Blood 125: 2605-2614.

Eijkelenboom A, Mokry M, de Wit E, Smits LM, Polderman PE, van Triest $\mathrm{MH}$, van Boxtel R, Schulze A, de Laat W, Cuppen E, et al. 2014. Genome-wide analysis of FOXO3 mediated transcription regulation through RNA polymerase II profiling. Mol Syst Biol 9: 638.

The ENCODE Project Consortium. 2012. An integrated encyclopedia of DNA elements in the human genome. Nature 489: 57-74.

Fahl SP, Crittenden RB, Allman D, Bender TP, Fahl SP, Crittenden RB, Allman D, Bender TP. 2009. c-Myb is required for Pro-B cell differentiation. J Immunol 183: 5582-5592.

Fiore C, Cohen BA. 2016. Interactions between pluripotency factors specify cis-regulation in embryonic stem cells. Genome Res 26: 778-786.

Freire-Pritchett P, Schoenfelder S, Várnai C, Steven W. 2017. Global reorganisation of cis-regulatory units upon lineage commitment of human embryonic stem cells. eLife 6: e21926. doi: 10.7554/eLife.21926.

Fullwood MJ, Liu MH, Pan YF, Liu J, Xu H, Mohamed YB, Orlov YL, Velkov S, Ho A, Mei PH, et al. 2009. An oestrogen-receptor- $\alpha$-bound human chromatin interactome. Nature 462: $58-64$

Ghavi-Helm Y, Klein FA, Pakozdi T, Ciglar L, Noordermeer D, Huber W, Furlong EEM. 2014. Enhancer loops appear stable during development and are associated with paused polymerase. Nature 512: $96-100$.

Greig KT, De Graaf CA, Murphy JM, Carpinelli MR, Heng S, Pang M, Frampton J, Kile BT, Hilton DJ, Nutt SL. 2010. Critical roles for c-Myb in lymphoid priming and early B-cell development. Blood 115: 2796-2806.

Heintzman ND, Stuart RK, Hon G, Fu Y, Ching CW, Hawkins RD, Barrera LO, Van Calcar S, Qu C, Ching KA, et al. 2007. Distinct and predictive chromatin signatures of transcriptional promoters and enhancers in the human genome. Nat Genet 39: 311-318.

Heintzman ND, Hon GC, Hawkins RD, Kheradpour P, Stark A, Harp LF, Ye Z, Lee LK, Stuart RK, Ching CW, et al. 2009. Histone modifications at human enhancers reflect global cell-type-specific gene expression. Nature 459: 108-112.

Hnisz D, Abraham BJ, Lee TI, Lau A, Saint-André V, Sigova AA, Hoke HA, Young RA. 2013. Super-enhancers in the control of cell identity and disease. Cell 155: 934-947.

Hnisz D, Day DS, Young RA. 2016. Insulated neighborhoods: structural and functional units of mammalian gene control. Cell 167: 1188-1200.

Hollenhorst PC, McIntosh LP, Graves BJ. 2011. Genomic and biochemical insights into the specificity of ETS transcription factors. Annu Rev Biochem 80: 437-471.

Ing-Simmons E, Seitan VC, Faure AJ, Flicek P, Carroll T, Dekker J, Fisher AG, Lenhard B, Merkenschlager M. 2015. Spatial enhancer clustering and regulation of enhancer-proximal genes by cohesin. Genome Res 25: 504-513.

Inoue F, Ahituv N. 2015. Genomics decoding enhancers using massively parallel reporter assays. Genomics 106: 159-164.

Jin F, Li Y, Dixon JR, Selvaraj S, Ye Z, Lee AY, Yen CA, Schmitt AD, Espinoza CA, Ren B. 2013. A high-resolution map of the three-dimensional chromatin interactome in human cells. Nature 503: 290-294.

Khan A, Zhang X. 2016. dbSUPER: a database of super-enhancers in mouse and human genome. Nucleic Acids Res 44: D164-D171.

Kimura S, Roberts AW, Metcalf D, Alexander WS. 1998. Hematopoietic stem cell deficiencies in mice lacking c-Mpl, the receptor for thrombopoietin. Proc Natl Acad Sci 95: 1195-1200.

Le Dily F, Baù D, Pohl A, Vicent GP, Serra F, Soronellas D, Castellano G, Wright RH, Ballare C, Filion G, et al. 2014. Distinct structural transitions of chromatin topological domains correlate with coordinated hormoneinduced gene regulation. Genes Dev 28: 2151-2162.

Levo M, Segal E. 2014. In pursuit of design principles of regulatory sequences. Nat Rev Genet 15: 453-468.

Li W, Notani D, Rosenfeld MG. 2016. Enhancers as non-coding RNA transcription units: recent insights and future perspectives. Nat Rev Genet 17: 207-223.

Lieberman-Aiden E, Van Berkum NL, Williams L, Imakaev M, Ragoczy T, Telling A, Amit I, Lajoie BR, Sabo PJ, Dorschner MO, et al. 2009. Comprehensive mapping of long-range interactions reveals folding principles of the human genome. Science 326: 289-293.

Lieu YK, Reddy EP. 2009. Conditional c-myb knockout in adult hematopoietic stem cells leads to loss of self-renewal due to impaired proliferation and accelerated differentiation. Proc Natl Acad Sci 106: 21689-21694.

Lin YC, Benner C, Mansson R, Heinz S, Miyazaki K, Miyazaki M, Chandra V, Bossen C, Glass CK, Murre C. 2012. Global changes in the nuclear positioning of genes and intra- and interdomain genomic interactions that orchestrate B cell fate. Nat Immunol 13: 1196-1204.
Liu YR, Laghari ZA, Novoa CA, Hughes J, Webster JRM, Goodwin PE, Wheatley SP, Scotting PJ. 2014. Sox2 acts as a transcriptional repressor in neural stem cells. BMC Neurosci 15: 95.

Lok S, Kaushansky K, Holly RD, Kuijper JL, Lofton-Day CE, Oort PJ, Grant FJ, Heipel MD, Burkhead SK, Kramer JM. 1994. Cloning and expression of murine thrombopoietin cDNA and stimulation of platelet production in vivo. Nature 369: 565-568.

Lun ATL, Smyth GK. 2015. csaw: a bioconductor package for differential binding analysis of ChIP-seq data using sliding windows. Nucleic Acids Res 44: e45. doi: 10.1093/nar/gkv1191.

Markenscoff-Papadimitriou E, Allen WE, Colquitt BM, Goh T, Murphy KK, Monahan K, Mosley CP, Ahituv N, Lomvardas S. 2014. Enhancer interaction networks as a means for singular olfactory receptor expression. Cell 159: 543-557.

McLean CY, Bristor D, Hiller M, Clarke SL, Schaar BT, Lowe CB, Wenger AM, Bejerano G. 2010. GREAT improves functional interpretation of cis-regulatory regions. Nat Biotechnol 28: 495-501.

Melo CA, Drost J, Wijchers PJ, van de Werken H, de Wit E, Oude Vrielink JA, Elkon R, Melo SA, Léveillé N, Kalluri R, et al. 2013. eRNAs are required for p53-dependent enhancer activity and gene transcription. Mol Cell 49: 524-535.

Méthot N, Basler K. 1999. Hedgehog controls limb development by regulating the activities of distinct transcriptional activator and repressor forms of Cubitus interruptus. Cell 96: 819-831.

Montavon T, Soshnikova N, Mascrez B, Joye E, Thevenet L, Splinter E, De Laat W, Spitz F, Duboule D. 2011. A regulatory archipelago controls Hox genes transcription in digits. Cell 147: 1132-1145.

Nayak A, Glöckner-Pagel J, Vaeth M, Schumann JE, Buttmann M, Bopp T, Schmitt E, Serfling E, Berberich-Siebelt F. 2009. Sumoylation of the tran scription factor NFATc1 leads to its subnuclear relocalization and interleukin-2 repression by histone deacetylase. I Biol Chem 284: 10935-10946.

Nishikii H, Kanazawa Y, Umemoto T, Goltsev Y, Matsuzaki Y, Matsushita K, Yamato M, Nolan GP, Negrin R, Chiba S. 2015. Unipotent megakaryopoietic pathway bridging hematopoietic stem cells and mature megakaryocytes. Stem Cells 33: 2196-2207.

Nora EP, Lajoie BR, Schulz EG, Giorgetti L, Okamoto I, Servant N, Piolot T, van Berkum NL, Meisig J, Sedat J, et al. 2012. Spatial partitioning of the regulatory landscape of the $\mathrm{X}$-inactivation centre. Nature 485: 381-385.

Nora EP, Goloborodko A, Valton AL, Gibcus JH, Uebersohn A, Abdennur N Dekker J, Mirny LA, Bruneau BG. 2017. Targeted degradation of CTCF decouples local insulation of chromosome domains from genomic compartmentalization. Cell 169: 930-944.e22.

Park HJ, Li J, Hannah R, Biddie S, Leal-Cervantes AI, Flores D, Cruz S, Sexl V, Göttgens B, Green AR. 2015. Cytokine-induced megakaryocytic differentiation is regulated by genome-wide loss of a uSTAT transcriptional program. EMBO J 35: 580-594.

Parker SC, Stitzel ML, Taylor DL, Orozco JM, Erdos MR, Akiyama JA, van Bueren KL, Chines PS, Narisu N, Black BL, et al. 2013. Chromatin stretch enhancer states drive cell-specific gene regulation and harbor human disease risk variants. Proc Natl Acad Sci 110: 17921-17926.

Phillips-Cremins JE, Sauria ME, Sanyal A, Gerasimova TI, Lajoie BR, Bell JS, Ong CT, Hookway TA, Guo C, Sun Y, et al. 2013. Architectural protein subclasses shape 3D organization of genomes during lineage commitment. Cell 153: 1281-1295.

Pinto do Ó P, Kolterud Å, Carlsson L. 1998. Expression of the LIM-homeobox gene $L H 2$ generates immortalized Steel factor-dependent multipotent hematopoietic precursors. EMBO J 17: 5744-5756.

Qian H, Buza-Vidas N, Hyland CD, Jensen CT, Antonchuk J, Månsson R, Thoren LA, Ekblom M, Alexander WS, Jacobsen SEW. 2007. Critica role of thrombopoietin in maintaining adult quiescent hematopoietic stem cells. Cell Stem Cell 1: 671-684.

Rao SS, Huang SC, Glenn B, Hilaire S, Casellas R, Lander ES, Aiden EL. 2017. Cohesin loss eliminates all loop domains. Cell 171: 305-309.

Reiter F, Wienerroither S, Stark A. 2017. Combinatorial function of transcription factors and cofactors. Curr Opin Genet Dev 43: 73-81.

Rieger MA, Schroeder T. 2012. Hematopoiesis. Cold Spring Harb Perspect Biol 4: $\mathrm{a} 008250$

Ruijtenberg S, van den Heuvel S. 2016. Coordinating cell proliferation and differentiation: antagonism between cell cycle regulators and cell typespecific gene expression. Cell Cycle 15: 196-212.

Sahlén P, Abdullayev I, Ramsköld D, Matskova L, Rilakovic N, Lötstedt B, Albert TJ, Lundeberg J, Sandberg R. 2015. Genome-wide mapping of promoter-anchored interactions with close to single-enhancer resolution. Genome Biol 16: 156.

Sánchez-Tilló E, De Barrios O, Valls E, Darling DS, Castells A, Postigo A. 2015. ZEB1 and TCF4 reciprocally modulate their transcriptional activities to regulate Wnt target gene expression. Oncogene 34: 5760-5770.

Schoenfelder S, Furlan-Magaril M, Mifsud B, Tavares-Cadete F, Sugar R, Javierre BM, Nagano T, Katsman Y, Sakthidevi M, Wingett SW, et al.

\section{Genome Research}

www.genome.org 
2015. The pluripotent regulatory circuitry connecting promoters to their long-range interacting elements. Genome Res 25: 582-597.

Schwarzer W, Abdennur N, Goloborodko A, Pekowska A, Fudenberg G, LoeMie Y, Fonseca NA, Huber W, Haering CH, Mirny L, et al. 2017. Two independent modes of chromatin organization revealed by cohesin removal. Nature 551: $51-56$

Servant N, Varoquaux N, Lajoie BR, Viara E, Chen CJ, Vert JP, Heard E, Dekker J, Barillot E. 2015. HiC-Pro: an optimized and flexible pipeline for Hi-C data processing. Genome Biol 16: 259. doi: 10.1186/s13059015-0831-x.

Sexton T, Yaffe E, Kenigsberg E, Bantignies F, Leblanc B, Hoichman M, Parrinello H, Tanay A, Cavalli G. 2012. Three-dimensional folding and functional organization principles of the Drosophila genome. Cell 148: 458-472.

Sharrocks AD. 2001. The ETS-domain transcription factor family. Nat Rev Mol Cell Biol 2: 827-837.

Shlyueva D, Stampfel G, Stark A. 2014. Transcriptional enhancers: from properties to genome-wide predictions. Nat Rev Genet 15: 272-286.

Smith E, Shilatifard A. 2014. Enhancer biology and enhanceropathies. Nat Struct Mol Biol 21: 210-219.

Solar GP, Kerr WG, Zeigler FC, Hess D, Donahue C, de Sauvage FJ, Eaton DL. 1998. Role of c-mpl in early hematopoiesis. Blood 92: 4-10.

Souroullas GP, Salmon JM, Sablitzky F, Curtis DJ, Goodell MA. 2009. Adult hematopoietic stem and progenitor cells require either $L y l 1$ or $S c l$ for survival. Cell Stem Cell 4: 180-186.

Spitz F, Furlong EE. 2012. Transcription factors: from enhancer binding to developmental control. Nat Rev Genet 13: 613-626.

Tibshirani R. 1996. Regression selection and shrinkage via the lasso. J R Stat Soc B 58: 267-288.

Vahedi G, Kanno Y, Furumoto Y, Jiang K, Parker SC, Erdos MR, Davis SR, Roychoudhuri R, Restifo NP, Gadina M, et al. 2015. Super-enhancers delineate disease-associated regulatory nodes in T cells. Nature 520: 558-562.
White MA, Kwasnieski JC, Myers CA, Shen SQ, Corbo JC, Cohen BA. 2016. A simple grammar defines activating and repressing cis-regulatory elements in photoreceptors. Cell Rep 17: 1247-1254.

Whyte WA, Orlando DA, Hnisz D, Abraham BJ, Lin CY, Kagey MH, Rahl PB, Lee TI, Young RA. 2013. Master transcription factors and mediator establish super-enhancers at key cell identity genes. Cell 153: 307-319.

Wilson NK, Foster SD, Wang X, Knezevic K, Schütte J, Kaimakis P, Chilarska PM, Kinston S, Ouwehand WH, Dzierzak E, et al. 2010. Combinatorial transcriptional control in blood stem/progenitor cells: genome-wide analysis of ten major transcriptional regulators. Cell Stem Cell 7: 532-544.

Wilson NK, Schoenfelder S, Hannah R, Sánchez Castillo M, Schutte J, Ladopoulos V, Mitchelmore J, Goode DK, Calero-Nieto FJ, Moignard $\mathrm{V}$, et al. 2016. Integrated genome-scale analysis of the transcriptional regulatory landscape in a blood stem/progenitor cell model. Blood 127: e12-e23.

Wutz G, Várnai C, Nagasaka K, Cisneros DA, Stocsits RR, Tang W, Schoenfelder S, Jessberger G, Muhar M, Hossain MJ, et al. 2017. Topologically associating domains and chromatin loops depend on cohesin and are regulated by CTCF, WAPL, and PDS5 proteins. EMBO J 36: 3573-3599.

Yoshihara H, Arai F, Hosokawa K, Hagiwara T, Takubo K, Nakamura Y, Gomei Y, Iwasaki H, Matsuoka S, Miyamoto K, et al. 2007. Thrombopoietin/MPL signaling regulates hematopoietic stem cell quiescence and interaction with the osteoblastic niche. Cell Stem Cell 1: 685-697.

Zhan Y, Mariani L, Barozzi I, Schulz EG, Bluthgen N, Stadler M, Tiana G, Giorgetti L. 2017. Reciprocal insulation analysis of Hi-C data shows that TADs represent a functionally but not structurally privileged scale in the hierarchical folding of chromosomes. Genome Res 27: 479-490.

Received July 6, 2017; accepted in revised form January 26, 2018. 


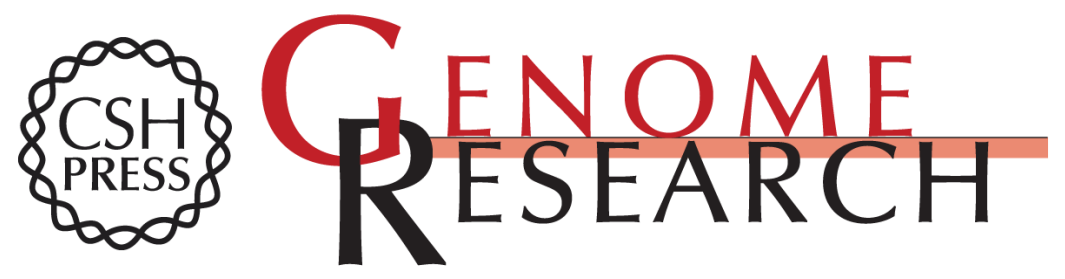

\section{Thrombopoietin signaling to chromatin elicits rapid and pervasive epigenome remodeling within poised chromatin architectures}

Federico Comoglio, Hyun Jung Park, Stefan Schoenfelder, et al.

Genome Res. 2018 28: 295-309 originally published online February 2, 2018

Access the most recent version at doi:10.1101/gr.227272.117

Supplemental Material

References

Open Access

Creative Commons License

Email Alerting Service
http://genome.cshlp.org/content/suppl/2018/02/14/gr.227272.117.DC1

This article cites 92 articles, 24 of which can be accessed free at: http://genome.cshlp.org/content/28/3/295.full.html\#ref-list-1

Freely available online through the Genome Research Open Access option.

This article, published in Genome Research, is available under a Creative Commons License (Attribution 4.0 International), as described at http://creativecommons.org/licenses/by/4.0/.

Receive free email alerts when new articles cite this article - sign up in the box at the top right corner of the article or click here.

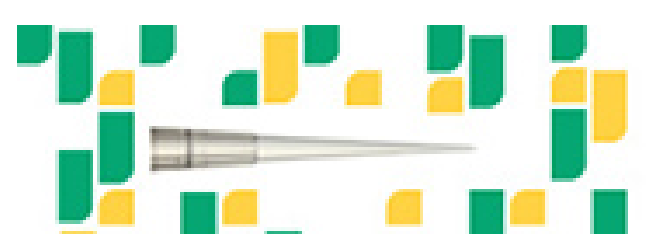

Focused on your science.

J⿹勹口

SCIENTIFIC

suos or seisnes

To subscribe to Genome Research go to: https://genome.cshlp.org/subscriptions 